\title{
The three-dimensional Fueter equation and divergence-free frames
}

\section{Journal Article}

\section{Author(s):}

Salamon, Dietmar

Publication date:

2013-04

Permanent link:

https://doi.org/10.3929/ethz-b-000067021

Rights / license:

In Copyright - Non-Commercial Use Permitted

Originally published in:

Abhandlungen aus dem Mathematischen Seminar der Universität Hamburg 83(1), https://doi.org/10.1007/s12188-013-0075-1 


\title{
The three-dimensional Fueter equation and divergence-free frames
}

\author{
Dietmar Salamon
}

Received: 20 July 2012 / Published online: 5 March 2013

(C) Mathematisches Seminar der Universität Hamburg and Springer-Verlag Berlin Heidelberg 2013

\begin{abstract}
This paper extends hyperkähler Floer theory with flat target manifolds from the case where the source is a 3-sphere or 3-torus, equipped with a standard frame, to the case where the source is a general closed orientable 3-manifold, equipped with a regular divergence-free frame. Regular divergence-free frames are characterized by the nonexistence of nonconstant solutions to the unperturbed linear Fueter equation. They form a dense open subset of the space of all divergence-free frames. A gauged version of the Fueter equation is introduced, which unifies various geometric equations in gauge theory.
\end{abstract}

Keywords (Gauged) Fueter equation · Divergence-free frames · Hyperkähler Floer theory

Mathematics Subject Classification 53D40

\section{Introduction}

The equation in the title was introduced by Rudolph Fueter in his study of analytic functions of one quaternionic variable in the 1930's [16, 17]. The three-dimensional reduction of the Fueter equation carries over to functions $f: M \rightarrow X$ from any three-manifold $M$ (equipped with a volume form and a global divergence-free frame $v_{1}, v_{2}, v_{3}$ ) to any hyperkähler manifold $X$ (with complex structures $J_{1}, J_{2}, J_{3}$ ). It has the form

$$
J_{1} \partial_{v_{1}} f+J_{2} \partial_{v_{2}} f+J_{3} \partial_{v_{3}} f=\nabla H(f) .
$$

The function $H: M \times X \rightarrow \mathbb{R}$ determines a zeroth order perturbation. There is a natural analogy between the solutions of (1) and periodic orbits of Hamiltonian systems in a symplectic manifold. The solutions of (1) are critical points of an action functional, there is a

Communicated by J. Latschev.

Partially supported by the Swiss National Science Foundation Grant 200021-127136.

D. Salamon $(\varangle)$

Department of Mathematics, ETHZ, 8092 Zurich, Switzerland

e-mail: salamon@math.ethz.ch 
Floer type theory for this functional, and an analogue of the Arnold conjecture holds in favorable cases. For flat target manifolds and standard frames on $S^{3}$ and $\mathbb{T}^{3}$ hyperkähler Floer theory was developed in $[23,24]$ and the Arnold conjecture was derived as a corollary. With different methods the hyperkähler Arnold conjecture was extended by Ginzburg-Hein [19, 20] to a more general setting.

The starting point for the present paper was the question under which condition the hyperkähler Floer theory in $[23,24]$ extends to general three-manifolds and divergencefree frames. The key point is an estimate which asserts that the $L^{2}$-norm of the sum $\not \partial_{v} f:=J_{1} \partial_{v_{1}} f+J_{2} \partial_{v_{2}} f+J_{3} \partial_{v_{3}} f$ controls the $L^{2}$ norm of $d f$ (Lemma 4.2). Such an estimate holds if and only if the linear Fueter equation with target space $X=\mathbb{H}$ (the quaternions) and zero Hamiltonian has no nonconstant solutions. If nonconstant solutions do exist, compactness fails for the solutions of equation (25). Call a divergence-free frame $v$ regular if every solution $f: M \rightarrow \mathbb{H}$ of the linear Fueter equation $\not_{v} f=0$ is constant and call it singular otherwise. Then the hyperkähler Floer theory with flat target manifolds carries over to all regular divergence-free frames and the hyperkähler Arnold conjecture holds in this case.

Section 2 discusses the space $\mathcal{V}$ of divergence-free frames on a closed three-manifold and the linear Fueter operator. It is shown that the set $\mathcal{V}^{\text {reg }}$ of regular divergence-free frames is open and dense in $\mathcal{V}$ (Lemma 2.3), that the set $\mathcal{V}_{1}$ of divergence-free frames $v \in \mathcal{V}$ with $\operatorname{dim}\left(\operatorname{ker} \not \partial_{v}\right)=8$ is a codimension one submanifold of $\mathcal{V}$ (Lemma 2.4), and the topology of $\mathcal{V}$ is examined (Lemma 2.7). Section 3 discusses several examples. It is shown that singular divergence-free frames exist on $M=S^{3}$ and that the standard frame on $M=S^{2} \times S^{1}$ is regular. Section 4 explains how the hyperkähler Floer theory of [23, 24] extends to regular divergence-free frames.

Section 6 introduces the gauged Fueter equation, associated to a G-action on $X$ generated by a hyperkähler moment map $\mu=\left(\mu_{1}, \mu_{2}, \mu_{3}\right): X \rightarrow \mathfrak{g}^{3}$. The four-dimensional version of this equation has the form

$$
\partial_{s} u+L_{u} \Phi-\not \partial_{A, v} u=0, \quad \partial_{s} A-d_{A} \Phi+* F_{A}=\sum_{i}\left(\mu_{i} \circ u\right) \pi^{*} \alpha_{i}
$$

for a principal G-bundle $P \rightarrow Y$, a G-equivariant function $u: \mathbb{R} \times P \rightarrow X$, and a G-connection $A(s)+\Phi(s) d s$ on $\mathbb{R} \times P$. This is analogous to the symplectic vortex equations $[2,28]$, and similar equations were studied by Haydys [22]. The Fueter equation in dimension four corresponds to $\mathrm{G}=1$, the Seiberg-Witten equations to $X=\mathbb{H}, \mathrm{G}=S^{1}$ (Sect. 7), Taubes' generalized Seiberg-Witten equations in [36] to $\mathrm{G}=S^{1}$, the PidstrigatchTyurin equations [31,32] to $X=\mathbb{H}, \mathrm{G}=\mathrm{Sp}(1)$, the instanton Floer equation to $X=$ \{point , and the Donaldson-Thomas $\mathrm{G}_{2}$-instantons on $Y=M \times \Sigma$ ( $\Sigma$ a hyperkähler surface $)$ appear when $X$ is taken to be the space of connections on $\Sigma$ and $\mathrm{G}$ the group of gauge transformations (Sect. 5).

Multiplying the right hand side in (2) by $\varepsilon^{-2}$ and taking the limit $\varepsilon \rightarrow 0$, one finds that equation (2) degenerates formally to the standard Fueter equation for functions with values in the hyperkähler quotient $X / / \mathrm{G}=\mu^{-1}(0) / \mathrm{G}$. This is reminiscent of the Atiyah-Floer conjecture [6]. In [38, 39] Walpuski carried out the adiabatic limit analysis to prove existence of $\mathrm{G}_{2}$-instantons.

Appendix A contains a proof of Gromov's theorem that the inclusion of the space of global divergence-free frames into the space of all frames is a homotopy equivalence. Appendix B discusses some functional analytic background about self-adjoint Fredholm operators that is needed in Sect. 2. 


\section{The Fueter equation}

Let $M$ be a closed oriented three-manifold and $\operatorname{dvol}_{M} \in \Omega^{3}(M)$ be a positive volume form. Then $M$ is parallelizable and a theorem of Gromov [21] asserts that every frame of $T M$ can be deformed to a divergence-free frame. The space of positive divergence-free frames will be denoted by

$$
\mathcal{V}:=\left\{\begin{array}{l|l}
\left(v_{1}, v_{2}, v_{3}\right) \in \operatorname{Vect}(M)^{3} & \begin{array}{l}
\mathcal{L}_{v_{i}} \operatorname{dvol}_{M}=0 \text { for } i=1,2,3 \\
\text { and } \operatorname{dvol}_{M}\left(v_{1}, v_{2}, v_{3}\right)>0 \text { on } M
\end{array}
\end{array}\right\} .
$$

Thus $\mathcal{V}$ is a subset of the space $\mathcal{F}$ of positive frames. Formally, there is an analogy between the inclusion $\mathcal{V} \hookrightarrow \mathcal{F}$ and the inclusion of the space of symplectic forms into the space of all nondegenerate 2 -forms. However, in contrast to the symplectic setting, the h-principle rules and the inclusion of $\mathcal{V}$ into $\mathcal{F}$ is a homotopy equivalence (see Theorem A.1).

Associated to every divergence-free frame $v=\left(v_{1}, v_{2}, v_{3}\right)$ is a self-adjoint Fredholm operator $\not_{v}$ defined as follows. Let $\mathbb{H}$ denote the space of quaternions and write the elements of $\mathbb{H}$ in the form $x=x_{0}+\mathbf{i} x_{1}+\mathbf{j} x_{2}+\mathbf{k} x_{3}$ with $x_{0}, x_{1}, x_{2}, x_{3} \in \mathbb{R}$. Define the complex structures $I_{1}, I_{2}, I_{3}$ and $J_{1}, J_{2}, J_{3}$ on $\mathbb{H}$ as right and left multiplication by $\mathbf{i}, \mathbf{j}, \mathbf{k}$, respectively. Thus, for $x \in \mathbb{H}$,

$$
\begin{aligned}
& J_{1} x:=\mathbf{i} x, \quad J_{2} x:=\mathbf{j} x, \quad J_{3} x:=\mathbf{k} x, \\
& I_{1} x:=-x \mathbf{i}, \quad I_{2} x:=-x \mathbf{j}, \quad I_{3} x:=-x \mathbf{k} .
\end{aligned}
$$

Thus the complex structures $J_{1}, J_{2}, J_{3}$ (respectively $I_{1}, I_{2}, I_{3}$ ) satisfy the Clifford relations. Given a divergence-free frame $v=\left(v_{1}, v_{2}, v_{3}\right) \in \mathcal{V}$ define the linear first order differential operator $\not \partial_{v}: \Omega^{0}(M, \mathbb{H}) \rightarrow \Omega^{0}(M, \mathbb{H})$ by

$$
\not \partial_{v} f:=J_{1} \partial_{v_{1}} f+J_{2} \partial_{v_{2}} f+J_{3} \partial_{v_{3}} f .
$$

This is the Fueter operator [16, 17]. It commutes with $I_{i}$ for $i=1,2,3$ and hence is equivariant under the right action of the quaternions on $\Omega^{0}(M, \mathbb{H})$. The divergence-free condition asserts that $\not \partial_{v}$ is symmetric with respect to the $L^{2}$ inner product on $\Omega^{0}(M, \mathbb{H})$ determined by the volume form and the standard inner product on $\mathbb{H}$. Denote this inner product by

$$
\langle f, g\rangle_{L^{2}}:=\int_{M}\langle f, g\rangle \mathrm{dvol}_{M}
$$

The notation (5) extends to any triple of divergence-free vector fields and still defines a symmetric operator. It is self-adjoint, whenever $v$ is also a frame.

Lemma 2.1 Let $v=\left(v_{1}, v_{2}, v_{3}\right) \in \mathcal{V}$. Then $\not_{v}: W^{1,2}(M, \mathbb{H}) \rightarrow L^{2}(M, \mathbb{H})$ is a symmetric operator and, for every $f \in L^{2}(M, \mathbb{H})$,

$$
\sup _{0 \neq g \in \Omega^{0}(M, \mathbb{H})} \frac{\left|\left\langle f, \not \partial_{v} g\right\rangle_{L^{2}}\right|}{\|g\|_{L^{2}}}<\infty \quad \Longleftrightarrow \quad f \in W^{1,2}(M, \mathbb{H}) .
$$

Proof That the operator is symmetric is obvious. To prove (6), define the divergence-free vector fields $w_{1}, w_{2}, w_{3} \in \operatorname{Vect}(M)$ by

$$
w_{1}:=\left[v_{2}, v_{3}\right], \quad w_{2}:=\left[v_{3}, v_{1}\right], \quad w_{3}:=\left[v_{1}, v_{2}\right] .
$$


(Here and throughout I use the sign convention $\mathcal{L}_{[u, v]}=-\left[\mathcal{L}_{u}, \mathcal{L}_{v}\right]$ for the Lie bracket of two vector fields $u, v \in \operatorname{Vect}(M)$.) Then

$$
\not_{v} \not_{v}=-\mathcal{L}_{v}-\not_{w}, \quad \mathcal{L}_{v}:=\sum_{i=1}^{3} \partial_{v_{i}} \partial_{v_{i}} .
$$

Hence, taking $g=\not{ }_{v} h$ in (6), we find

$$
\sup _{g \neq 0} \frac{\left|\left\langle f, \not \partial_{v} g\right\rangle_{L^{2}}\right|}{\|g\|_{L^{2}}}<\infty \Longrightarrow \sup _{h \neq 0} \frac{\left|\left\langle f, \mathcal{L}_{v} h\right\rangle_{L^{2}}\right|}{\|h\|_{W^{1,2}}}<\infty .
$$

Now it follows from standard elliptic regularity for the second order operator $\mathcal{L}_{v}$ that $f \in$ $W^{1,2}(M, \mathbb{H})$. This proves Lemma 2.1 .

By Lemma 2.1, the operator $\not_{v}: W^{1,2}(M, \mathbb{H}) \rightarrow L^{2}(M, \mathbb{H})$ satisfies condition (i) in Lemma B.1 and hence is a self-adjoint index zero Fredholm operator for every $v \in \mathcal{V}$. Its kernel consists of smooth functions, by elliptic regularity, and contains the constant functions. The dimension of the kernel is divisible by four.

Definition 2.2 A divergence-free frame $v \in \mathcal{V}$ is called regular if every solution $f: M \rightarrow \mathbb{H}$ of the linear Fueter equation $\not_{v} f=0$ is constant. Otherwise $v$ is called singular. The set of regular (respectively singular) divergence-free frames is denoted by $\mathcal{V}^{\text {reg }}$ (respectively $\left.\mathcal{V}^{\text {sing }}\right)$.

Lemma 2.3 The set $\mathcal{V}^{\text {reg }}$ is open and dense in $\mathcal{V}$.

Proof Fix a divergence-free frame $v \in \mathcal{V}$ and let $w=\left(w_{1}, w_{2}, w_{3}\right)$ be as in (7). Denote by $L_{0}^{2}(M, \mathbb{H}) \subset L^{2}(M, \mathbb{H})$ and $W_{0}^{1,2}(M, \mathbb{H}) \subset W^{1,2}(M, \mathbb{H})$ the spaces of functions with mean value zero and consider the operator family

$$
D(s):=\not_{v+s w}: W_{0}^{1,2}(M, \mathbb{H}) \rightarrow L_{0}^{2}(M, \mathbb{H}) .
$$

The path $s \mapsto D(s)$ is differentiable with derivative $\dot{D}(0)=\not \not_{w}$. By (8),

$$
\int_{M}\left\langle f, \not_{w} g\right\rangle \mathrm{dvol}_{M}=\int_{M} \sum_{i=1}^{3}\left\langle\partial_{v_{i}} f, \partial_{v_{i}} g\right\rangle \mathrm{dvol}_{M}
$$

for all $f, g \in W_{0}^{1,2}(M, \mathbb{H})$ with $\not \partial_{v} f=\not_{v} g=0$. Hence the path $s \mapsto D(s)$ has a positive definite crossing form at $s=0$. Hence, by Lemma B.2, the operator $\not_{v+s w}: W_{0}^{1,2}(M, \mathbb{H}) \rightarrow$ $L_{0}^{2}(M, \mathbb{H})$ is bijective for $s \neq 0$ sufficiently small and so $v+s w \in \mathcal{V}^{\text {reg }}$ for small $s \neq 0$. Thus $\mathcal{V}^{\text {reg }}$ is dense in $\mathcal{V}$. That $\mathcal{V}^{\text {reg }}$ is an open subset of $\mathcal{V}$ is obvious.

The set of divergence-free frames decomposes as the disjoint union

$$
\mathcal{V}=\bigcup_{k=0}^{\infty} \mathcal{V}_{k}, \quad \mathcal{V}_{k}:=\left\{v \in \mathcal{V} \mid \operatorname{dim}\left(\operatorname{ker} \not_{v}\right)=4(k+1)\right\}
$$

In this notation $\mathcal{V}^{\text {reg }}=\mathcal{V}_{0}$ and $\mathcal{V}^{\text {sing }}=\bigcup_{k=1}^{\infty} \mathcal{V}_{k}$. By Lemma $2.3, \mathcal{V}_{0}$ is a dense open subset of $\mathcal{V}$. 
Lemma $2.4 \mathcal{V}_{1}$ is a codimension one Fréchet submanifold of $\mathcal{V}$.

Proof Let $\Omega_{0}^{0}(M, \mathbb{H})$ be the space of smooth functions $f: M \rightarrow \mathbb{H}$ with mean value zero and define

$$
\mathscr{B}:=\left\{(v, f) \in\left(\mathcal{V}_{0} \cup \mathcal{V}_{1}\right) \times\left.\Omega_{0}^{0}(M, \mathbb{H})\left|\int_{M}\right| f\right|^{2} \mathrm{dvol}_{M}=1\right\} .
$$

Since $\mathcal{V}_{0} \cup \mathcal{V}_{1}$ is an open subset of the Fréchet space of triples of divergence-free vector fields, $\mathscr{B}$ is a Fréchet manifold with tangent spaces

$$
T_{(v, f)} \mathcal{B}=\left\{\begin{array}{l|l}
(\widehat{v}, \widehat{f}) \in \operatorname{Vect}(M)^{3} \times \Omega_{0}^{0}(M, \mathbb{H}) & \begin{array}{l}
\mathcal{L}_{\widehat{v}_{i}} \mathrm{dvol}_{M}=0, \\
\int_{M}\langle f, \widehat{f}\rangle \operatorname{dvol}_{M}=0
\end{array}
\end{array} .\right.
$$

This space carries a free action of the Lie group $\operatorname{Sp}(1)$ (the unit quaternions) by $x_{*}(v, f):=$ $\left(v, x_{0} f+\sum_{i=1}^{3} x_{i} I_{i} f\right)$ for $x=x_{0}+\mathbf{i} x_{1}+\mathbf{j} x_{2}+\mathbf{k} x_{3} \in \operatorname{Sp}(1)$. So does the total space of the vector bundle $\mathscr{E} \rightarrow \mathscr{B}$ with fibers

$$
\mathcal{E}_{(v, f)}:=\left\{h \in \Omega_{0}^{0}(M, \mathbb{H}) \mid \int_{M}\left\langle h, I_{i} f\right\rangle \operatorname{dvol}_{M}=0 \text { for } i=1,2,3\right\} .
$$

This bundle has a natural $\mathrm{Sp}(1)$-equivariant section

$$
\S: \mathscr{B} \rightarrow \mathcal{E}, \quad \&(v, f):=\not \partial_{v} f .
$$

The intrinsic differential of $s$ at a zero $(v, f) \in \mathscr{B}$ is the linear operator

$$
\mathcal{D}_{(v, f)}: T_{(v, f)} \mathcal{B} \rightarrow \mathcal{E}_{(v, f)}, \quad \mathcal{D}_{(v, f)}(\widehat{v}, \widehat{f}):=\not \not_{v} \widehat{f}+\not \partial_{\widehat{v}} f .
$$

The kernel of the operator $\partial_{v}: \Omega_{0}^{0}(M, \mathbb{H}) \rightarrow \Omega_{0}^{0}(M, \mathbb{H})$ is equal to its cokernel, has dimension four, and is spanned by $f, I_{1} f, I_{2} f, I_{3} f$ whenever $(v, f) \in \mathscr{B}$ and $\not_{v} f=0$. The summand $\widehat{f} \mapsto \not_{v} \widehat{f}$ in $\mathscr{D}_{(v, f)}$ is restricted to a codimension one subspace of $\Omega_{0}^{0}(M, \mathbb{H})$ (the $L^{2}$ orthgonal complement of $f$ ) while the target is restricted to the codimension three subspace $\mathcal{E}_{(v, f)}$ (the $L^{2}$ orthogonal complement of $I_{1} f, I_{2} f, I_{3} f$ ). Thus its kernel has dimension three, its cokernel has dimension one, and so its Fredholm index is two. Hence the projection

$$
\left\{(\widehat{v}, \widehat{f}) \in T_{(v, f)} \mathcal{B} \mid \not \partial_{v} \widehat{f}+\not \not_{\widehat{v}} f=0\right\} \rightarrow T_{v} \mathcal{V}:(\widehat{v}, \widehat{f}) \mapsto \widehat{v}
$$

is a Fredholm operator of Fredholm index two. (See [27, Lemma A.3.6].)

Let $(v, f) \in \mathscr{B}$ such that $\not_{v} f=0$. Then $\mathscr{D}_{(v, f)}$ is surjective. To see this, observe that $\mathscr{D}_{(v, f)}: T_{(v, f)} \mathscr{B} \rightarrow \mathcal{E}_{(v, f)}$ has a closed image, by standard elliptic theory, and hence it suffices to prove that the image is dense. Thus let $h \in L^{2}(M ; \mathbb{H})$ be a function with mean value zero, orthogonal to $I_{1} f, I_{2} f, I_{3} f$ (i.e. in the $L^{2}$ completion of $\varepsilon_{(v, f)}$ ), and $L^{2}$ orthogonal to the image of $\mathscr{D}_{(v, f)}$. Then

$$
\begin{aligned}
& \int_{M} h \operatorname{dvol}_{M}=0, \quad \int_{M}\left\langle h, I_{i} f\right\rangle \operatorname{dvol}_{M}=0 \quad \text { for } i=1,2,3, \\
& \int_{M}\left\langle h, \not \partial_{v} \widehat{f}\right\rangle \operatorname{dvol}_{M}=0 \quad \text { for } \widehat{f} \in \Omega_{0}^{0}(M, \mathbb{H}) \text { with } \int_{M}\langle f, \widehat{f}\rangle \operatorname{dvol}_{M}=0, \\
& \int_{M}\left\langle h, \not \partial_{\widehat{v}} f\right\rangle \operatorname{dvol}_{M}=0 \quad \text { for } \widehat{v} \in T_{v} \mathcal{V} .
\end{aligned}
$$


It follows from (12) and elliptic regularity that $h: M \rightarrow \mathbb{H}$ is smooth and that $\not \partial_{v} h-h_{0} \in \mathbb{R} f$ for some element $h_{0} \in \mathbb{H}$. Since $\not \partial_{v} f=0$ it follows that $\not \partial_{v} \not \partial_{v} h=0$ and hence $\not \partial_{v} h=0$. (Take the $L^{2}$-inner product with $h$ and integrate by parts.) Since the kernel of $\not_{v}: \Omega_{0}^{0}(M, \mathbb{H}) \rightarrow$ $\Omega_{0}^{0}(M, \mathbb{H})$ is spanned by $f, I_{1} f, I_{2} f, I_{3} f$ it follows from (11) that $h=\lambda f$ for some $\lambda \in \mathbb{R}$. Hence it follows from (13), with $\widehat{v}=w$ given by (7), and from (8) that

$$
0=\int_{M}\left\langle h, \partial_{w} f\right\rangle \operatorname{dvol}_{M}=\int_{M} \sum_{i=1}^{3}\left\langle\partial_{v_{i}} h, \partial_{v_{i}} f\right\rangle \operatorname{dvol}_{M}=\lambda \int_{M}|d f|^{2} \operatorname{dvol}_{M}
$$

Since $f$ is nonconstant, this implies $\lambda=0$ and hence $h=0$.

This shows that the operator $\mathcal{D}_{(v, f)}: T_{(v, f)} \mathscr{B} \rightarrow \mathcal{E}_{(v, f)}$ is surjective for all $(v, f) \in \mathscr{B}$ with $\not_{v} f=0$. Via Sobolev completion and the infinite-dimensional implicit function theorem, it follows that the set

$$
\mathcal{P}:=\left\{(v, f) \in \mathscr{B} \mid \not \partial_{v} f=0\right\}
$$

is a Fréchet submanifold of $\mathscr{B}$ with tangent spaces

$$
T_{(v, f)} \mathcal{P}=\left\{(\widehat{v}, \widehat{f}) \in T_{(v, f)} \mathscr{B} \mid \not \partial_{v} \widehat{f}+\not \not_{\widehat{v}} f=0\right\} .
$$

The group $\operatorname{Sp}(1)$ acts freely on $\mathcal{P}$ and the quotient space $\mathcal{P} / \mathrm{Sp}(1)$ is homeomorphic to $\mathcal{V}_{1}$ via the projection $\pi: \mathcal{P} \rightarrow \mathcal{V}$ defined by $\pi(v, f):=v$. The derivative of $\pi$ is the linear operator $d \pi(v, f): T_{(v, f)} \mathcal{P} \rightarrow T_{v} \mathcal{V}$ given by $d \pi(v, f)(\widehat{v}, \widehat{f})=\widehat{v}$. This is a Fredholm operator of index two (equation (10)) and it has a three-dimensional kernel. Hence its cokernel has dimension one. This implies, again via suitable Sobolev completions, that the map $\pi: \mathcal{P} \rightarrow \mathcal{V}$ descends to an injective immersion $\iota: \mathcal{P} / \mathrm{Sp}(1) \rightarrow \mathcal{V}$ with image $\mathcal{V}_{1}$ and derivative of Fredholm index -1 . The immersion $\iota$ is obviously proper (compact subsets of $\mathcal{V}_{1}$ have compact preimages in $\mathcal{P} / \mathrm{Sp}(1)$ ) and hence $\iota$ is an embedding. This proves Lemma 2.4.

Conjecture 2.5 $\mathcal{V}_{k}$ is a Fréchet submanifold of $\mathcal{V}$ of codimension $2 k^{2}-k$.

Conjecture 2.5 is motivated by an analogous result for quaternionic-hermitian matrices and by Lemma 2.4. If the conjecture is true, then every path $s \mapsto v^{s}$ in $\mathcal{V}$ with endpoints in $\mathcal{V}_{0}$ is homotopic, with fixed endpoints, to a path that is transverse to $\mathcal{V}_{1}$ and misses $\mathcal{V}_{k}$ for $k>1$. The resulting path $s \mapsto \not_{v^{s}}$ of self-adjoint Fredholm operators then has only regular crossings and its spectral flow is the intersection number with $\mathcal{V}_{1}$.

Remark 2.6 (Spectral flow) A loop of divergence-free frames with nonzero spectral flow, if it exists, has infinite order in $\pi_{1}(\mathcal{V})$. The existence of such a loop would prove that $\mathcal{V}^{\text {sing }} \neq \emptyset$. If the fundamental group of a connected component $\mathcal{V}^{\prime} \subset \mathcal{V}$ is finite and $\mathcal{V}^{\text {sing }} \cap \mathcal{V}^{\prime} \neq \emptyset$, then the spectral flow of a path with endpoints in $\mathcal{V}^{\text {reg }} \cap \mathcal{V}^{\prime}$ depends only on the endpoints, so $\mathcal{V}^{\text {reg }} \cap \mathcal{V}^{\prime}$ is disconnected. (See Example 3.3 below for $M=S^{3}$.)

The next lemma relates the topology of $\mathcal{V}$ to the topology of the group of gauge transformations

$$
g:=\operatorname{Map}(M, \operatorname{SO}(3))
$$

The identity component of $g$ will be denoted by

$$
g_{0}:=\{g: M \rightarrow \operatorname{SO}(3) \mid g \text { lifts to a degree zero map } \tilde{g}: M \rightarrow \operatorname{Sp}(1)\}
$$


and the group of based gauge transformations, associated to $p^{*} \in M$, by

$$
g^{*}:=\left\{g: M \rightarrow \operatorname{SO}(3) \mid g\left(p^{*}\right)=\mathbb{1}\right\} .
$$

Their intersection is the group

$$
g_{0}^{*}:=g_{0} \cap g^{*} \cong\left\{\widetilde{g}: M \rightarrow \operatorname{Sp}(1) \mid \operatorname{deg}(\widetilde{g})=0, \widetilde{g}\left(p^{*}\right)=1\right\} .
$$

Lemma 2.7 (i) $\mathcal{V}$ is homotopy equivalent to $\mathcal{G}$ and each connected component of $\mathcal{V}$ is homotopy equivalent to $\mathrm{g}_{0}^{*} \times \mathrm{SO}(3)$.

(ii) There is a short exact sequence

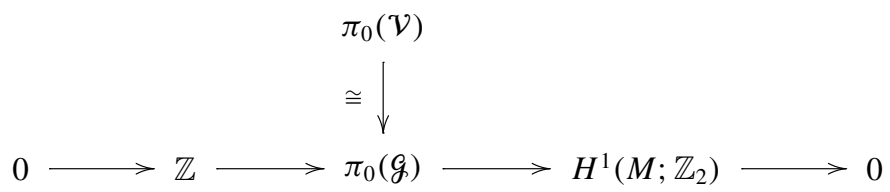

where the homomorphism $\mathbb{Z} \rightarrow \pi_{0}(g)$ assigns to an integer $d$ the homotopy class of maps $M \rightarrow \mathrm{SO}(3)$ that lift to degree-d maps $M \rightarrow \mathrm{Sp}(1)$, and the homomorphism $\pi_{0}(\mathcal{G})=$ $\pi_{0}\left(g^{*}\right) \rightarrow \operatorname{Hom}\left(\pi_{1}\left(M, p^{*}\right), \mathbb{Z}_{2}\right)=H^{1}\left(M ; \mathbb{Z}_{2}\right)$ assigns to a based gauge transformation $g: M \rightarrow \mathrm{SO}(3)$ the induced homomorphism $g_{*}: \pi_{1}\left(M, p^{*}\right) \rightarrow \pi_{1}(\mathrm{SO}(3), \mathbb{1})=\mathbb{Z}_{2}$.

(iii) Let $\mathcal{V}$ ' be a connected component of $\mathcal{V}$. Then

$$
\pi_{1}\left(\mathcal{V}^{\prime}\right) \cong \pi_{1}\left(G_{0}^{*}\right) \times \mathbb{Z}_{2}
$$

If $M=S^{3}$ then $\pi_{1}\left(\mathcal{V}^{\prime}\right) \cong \mathbb{Z}_{2} \times \mathbb{Z}_{2}$.

(iv) Let $M=S^{3}=\mathrm{Sp}(1) \subset \mathbb{H}$ be the unit sphere in the quaternions and denote by $\mathcal{V}^{\prime} \subset \mathcal{V}$ the connected component of the standard frame of $T M$. Then the inclusion

$$
\mathrm{SO}(3) \rightarrow \mathcal{V}^{\prime}: A \mapsto v_{A}=\left(v_{A, 1}, v_{A, 2}, v_{A, 3}\right),
$$

which assigns to a matrix $A=\left(a_{i j}\right)_{1 \leq i, j \leq 3} \in \mathrm{SO}(3)$ the divergence-free frame defined by $v_{A, i}(y):=a_{1 i} \mathbf{i} y+a_{2 i} \mathbf{j} y+a_{3 i} \mathbf{k} y$ for $y \in S^{3}$ and $i=1,2,3$, induces an isomorphism on rational homology.

Proof By Theorem A.1 the inclusion of $\mathcal{V}$ into the space $\mathcal{F}$ of positive global frames is a homotopy equivalence. So is the inclusion into $\mathcal{F}$ of the space of positive global orthonormal frames (associated to a Riemannian metric). The latter is homeomorphic to the gauge group $g$. Hence $\mathcal{V}$ is homotopy equivalent to $G$. An explicit homotopy equivalence from $\mathcal{V}$ to $g$ can be constructed by fixing a positive global frame of $T M$ and defining

$$
\mathcal{V} \rightarrow g: v \mapsto g_{v}:=A_{v}\left(A_{v}^{T} A_{v}\right)^{-1 / 2},
$$

where $A_{v}: M \rightarrow \mathrm{GL}^{+}\left(\mathbb{R}^{3}\right)$ is the gauge transformation relating $v$ to the reference frame. Now the map

$$
g \rightarrow g^{*} \times \mathrm{SO}(3): g \mapsto\left(g g\left(p^{*}\right)^{-1}, g\left(p^{*}\right)\right)
$$

is a homeomorphism and restricts to a homeomorphism $g_{0} \cong g_{0}^{*} \times \operatorname{SO}(3)$. This proves (i). 
Next observe that a based gauge transformation $g: M \rightarrow \mathrm{SO}(3)$ lifts to a map $\tilde{g}: M \rightarrow$ $\mathrm{Sp}(1)$ if and only if the induced map

$$
g_{*}: \pi_{1}\left(M, p^{*}\right) \rightarrow \pi_{1}(\mathrm{SO}(3), \mathbb{1})=\mathbb{Z}_{2}
$$

on fundamental groups is trivial. Hence the kernel of the map

$$
g^{*} \rightarrow \operatorname{Hom}\left(\pi_{1}\left(M, p^{*}\right), \mathbb{Z}_{2}\right)=H^{1}\left(M, \mathbb{Z}_{2}\right): g \mapsto g_{*}
$$

is isomorphic to the subgroup of all based gauge transformations that lift to maps $M \rightarrow S^{3}$. Hence exactness at $\mathbb{Z}$ and exactness at $\pi_{0}(g) \cong \pi_{0}\left(g^{*}\right)$ follow from the Hopf degree theorem. Exactness at $H^{1}\left(M ; \mathbb{Z}_{2}\right)$ follows by considering the subgroup of gauge transformations with values in the standard circle in $\mathrm{SO}(3)$. This proves (ii).

It follows immediately from (i) that $\pi_{1}\left(\mathcal{V}^{\prime}\right) \cong \pi_{1}\left(g_{0}^{*}\right) \times \mathbb{Z}_{2}$. If $M=S^{3}$ then $\pi_{1}\left(g_{0}^{*}\right) \cong$ $\pi_{4}\left(S^{3}\right) \cong \mathbb{Z}_{2}$ and hence $\pi_{1}\left(\mathcal{V}^{\prime}\right) \cong \mathbb{Z}_{2} \times \mathbb{Z}_{2}$. This proves (iii).

Assertion (iv) follows from (i) and a result of Donaldson and Kronheimer (Lemma 5.1.14 in [5]), which asserts that the group $g_{0}^{*} \cong\left(\Omega^{3} S^{3}\right)_{0}$ has the rational homology of a point. This proves Lemma 2.7.

Remark 2.8 (K-theory) The positive divergence-free frames $v \in \mathcal{V}$ parametrize the universal family of (self-adjoint) Fueter operators $\not \partial_{v}$. This family determines a K-theory class

$$
\operatorname{index}(\not \partial) \in K^{-1}(\mathcal{V})=K^{0}\left(S^{1} \times \mathcal{V}\right)
$$

(see Atiyah-Patodi-Singer [1]). Its Chern character determines an odd-dimensional cohomology class $\operatorname{ch}(\operatorname{index}(\not \partial)) \in H^{\text {odd }}(\mathcal{V} ; \mathbb{Q}$ ) (given by the spectral flow in degree one). When $M=S^{3}$ and $\mathcal{V}^{\prime}$ is the connected component of the standard frame, it follows from Lemma 2.7(iv) that the restriction of the class $\operatorname{ch}(\operatorname{index}(\not \partial))$ to $\mathcal{V}^{\prime}$ is determined by its pullback under the embedding $\mathrm{SO}(3) \rightarrow \mathcal{V}^{\prime}$ in (17). Since the image of this embedding is contained in $\mathcal{V}_{0}$ (see Example 3.2 below), the dimension of the kernel of $\not_{v}$ is constant along this embedding and hence its class in $K^{-1}(\mathrm{SO}(3))$ is trivial (see Ebert [9, Theorem 4.2.1]). Hence the Chern character of the K-theory class index $(\not \partial) \in K^{-1}\left(\mathcal{V}^{\prime}\right)$ vanishes in $H^{\text {odd }}\left(\mathcal{V}^{\prime} ; \mathbb{Q}\right)$, when $M=S^{3}$, and thus the K-theory class itself vanishes in $K^{-1}\left(\mathcal{V}^{\prime}\right)$ modulo torsion.

Remark 2.9 (Dual frame) Let $v \in \mathcal{V}$ and denote by $\alpha_{1}, \alpha_{2}, \alpha_{3} \in \Omega^{1}(M)$ the dual frame so that $\alpha_{i}\left(v_{j}\right)=\delta_{i j}$. Define a metric on $M$ by

$$
\langle u, v\rangle:=\sum_{i=1}^{3} \alpha_{i}(u) \alpha_{i}(v), \quad u, v \in T_{y} M .
$$

Then the vector fields $v_{1}, v_{2}, v_{3}$ form an orthonormal frame and the volume form is $\alpha_{1} \wedge$ $\alpha_{2} \wedge \alpha_{3}$. It does not, in general, agree with $\mathrm{dvol}_{M}$. They agree up to a constant factor if and only if the 2-forms $\alpha_{j} \wedge \alpha_{k}$ are closed. To see this, define $\lambda:=\operatorname{dvol}_{M}\left(v_{1}, v_{2}, v_{3}\right)$. Then $\iota\left(v_{i}\right) \mathrm{dvol}_{M}=\lambda \alpha_{j} \wedge \alpha_{k}$ for every cyclic permutation $i, j, k$ of $1,2,3$. If $\alpha_{j} \wedge \alpha_{k}$ is closed for $j \neq k$ it follows that $d \lambda \wedge \alpha_{j} \wedge \alpha_{k}=0$, hence $\partial_{v_{i}} \lambda=0$ for all $i$, and hence $\lambda$ is constant.

Remark 2.10 (Laplace-Beltrami operator) The pointwise norm of the differential $d f$ of a function $f: M \rightarrow \mathbb{H}$ with respect to the metric (19) is given by $|d f|^{2}=\sum_{i}\left|\partial_{v_{i}} f\right|^{2}$. If the 
function $\lambda:=\operatorname{dvol}_{M}\left(v_{1}, v_{2}, v_{3}\right)$ is constant, then $\mathcal{L}_{v}$ is the Laplace-Beltrami operator of the metric (19). Otherwise it is the composition of $d$ and its formal adjoint with respect to the $L^{2}$ inner products on functions and 1-forms associated to the pointwise inner products of the metric (19) and the volume form $\operatorname{dvol}_{M}$. It is then related to the Laplace-Beltrami operator by the formula

$$
\mathcal{L}_{v} f=-\frac{1}{\lambda} d^{*}(\lambda d f)=-d^{*} d f+\frac{1}{\lambda} \sum_{i}\left(\partial_{v_{i}} \lambda\right) \partial_{v_{i}} f
$$

for $f \in \Omega^{0}(M, \mathbb{H})$.

Definition 2.11 A divergence-free frame $\left(v_{1}, v_{2}, v_{3}\right) \in \mathcal{V}\left(M, \operatorname{dvol}_{M}\right)$ is called normal if $\operatorname{dvol}_{M}\left(v_{1}, v_{2}, v_{3}\right)=1$.

Lemma 2.12 Every positive regular (respectively singular) divergence-free frame $v \in$ $\mathcal{V}\left(M, \mathrm{dvol}_{M}\right)$ can be deformed through regular (respectively singular) divergence-free frames to a normal regular (respectively singular) divergence-free frame.

Proof Let $v \in \mathcal{V}^{\text {reg }}\left(M, \operatorname{dvol}_{M}\right)$ and $\lambda:=\operatorname{dvol}_{M}\left(v_{1}, v_{2}, v_{3}\right)$. Define

$$
\rho_{t}:=c_{t} \lambda^{t / 2} \operatorname{dvol}_{M}, \quad v_{i, t}:=c_{t}^{-1 / 3} \lambda^{-t / 2} v_{i}, \quad c_{t}:=\frac{\int_{M} \mathrm{dvol}_{M}}{\int_{M} \lambda^{t / 2} \mathrm{dvol}_{M}} .
$$

Then $v_{t}:=\left(v_{1, t}, v_{2, t}, v_{3, t}\right) \in \mathcal{V}\left(M, \rho_{t}\right)$ for every $t$. Second, $v_{t}$ is equal to $v$ for $t=0$ and is a normal frame for $t=1$. Third, $v_{t}$ is regular for every $t$, because $\operatorname{ker} \not_{v_{t}}=\operatorname{ker} \not_{v}$. Fourth $\rho_{0}=$ $\mathrm{dvol}_{M}$ and $\int_{M} \rho_{t}=\int_{M} \mathrm{dvol}_{M}$ for all $t$. By Moser isotopy, choose a smooth isotopy $[0,1] \rightarrow$ $\operatorname{Diff}(M): t \mapsto \phi_{t}$ such that $\phi_{0}=\mathrm{id}$ and $\phi_{t}^{*} \rho_{t}=\mathrm{dvol}_{M}$ for all $t$. The required isotopy of regular frames is $\phi_{t}^{*} v_{t} \in \mathcal{V}^{\mathrm{reg}}\left(M, \mathrm{dvol}_{M}\right), 0 \leq t \leq 1$. Namely, the frame $\phi_{t}^{*} v_{t}$ is regular for every $t$ because

$$
\operatorname{ker} \not_{\phi_{t}^{*} v_{t}}=\phi_{t}^{*} \operatorname{ker} \not_{v_{t}}=\phi_{t}^{*} \operatorname{ker} \not_{v}=0 .
$$

This proves Lemma 2.12 .

\section{Examples}

Fix a divergence-free frame $v \in \mathcal{V}\left(M, \operatorname{dvol}_{M}\right)$ and let $\alpha_{1}, \alpha_{2}, \alpha_{3} \in \Omega^{1}(M)$ be the dual frame (see Remark 2.9). For $i=1,2,3$ define $\omega_{i} \in \Omega^{2}(\mathbb{H})$ by $\omega_{i}=d x_{0} \wedge d x_{i}+d x_{j} \wedge d x_{k}$, where $i, j, k$ is a cyclic permutation of $1,2,3$. Let $f: M \rightarrow \mathbb{H}$ be a smooth map and abbreviate $|d f|^{2}:=\sum_{i}\left|\partial_{v_{i}} f\right|^{2}$. Then there is an energy identity

$$
\frac{1}{2} \int_{M}|d f|^{2} \operatorname{dvol}_{M}=\left.\frac{1}{2} \int_{M}|\not \partial f|\right|^{2} \operatorname{dvol}_{M}-\sum_{i} \int_{M} \alpha_{i} \wedge f^{*} \omega_{i} .
$$

This continues to hold for maps from $M$ to any hyperkähler manifold $X$.

Example 3.1 Consider the 3-torus $M=\mathbb{T}^{3}=\mathbb{R}^{3} / \mathbb{Z}^{3}$. Every linearly independent triple of constant vector fields $v_{1}, v_{2}, v_{3}$ is a regular divergence-free frame. Namely, the dual frame consists of closed 1-forms, hence the second term on the right in (20) vanishes for functions $f: M \rightarrow \mathbb{H}$, and hence every solution of the Fueter equation is constant. 
Example 3.2 Consider the 3 -sphere $M=S^{3} \subset \mathbb{H}$ with the volume form $\mathrm{dvol}_{M}=$ $y_{0} d y_{1} d y_{2} d y_{3}-y_{1} d y_{0} d y_{2} d y_{3}-y_{2} d y_{0} d y_{3} d y_{1}-y_{3} d y_{0} d y_{1} d y_{2}$ and the frame

$$
v_{1}(y)=\mathbf{i} y, \quad v_{2}(y)=\mathbf{j} y, \quad v_{3}(y)=\mathbf{k} y .
$$

The $v_{i}$ are divergence-free and $\operatorname{dvol}_{M}\left(v_{1}, v_{2}, v_{3}\right)=1$. The dual frame is

$$
\begin{aligned}
& \alpha_{1}=y_{0} d y_{1}-y_{1} d y_{0}+y_{2} d y_{3}-y_{3} d y_{2}, \\
& \alpha_{2}=y_{0} d y_{2}-y_{2} d y_{0}+y_{3} d y_{1}-y_{1} d y_{3}, \\
& \alpha_{3}=y_{0} d y_{3}-y_{3} d y_{0}+y_{1} d y_{2}-y_{2} d y_{1} .
\end{aligned}
$$

Note that $\left[v_{j}, v_{k}\right]=2 v_{i}$ and $d \alpha_{i}=2 \alpha_{j} \wedge \alpha_{k}=2 \iota\left(v_{i}\right) \operatorname{dvol}_{M}$ for every cyclic permutation $i, j, k$ of $1,2,3$. The energy identity (20) has the form

$$
\frac{1}{2} \int_{M}|d f|^{2} \operatorname{dvol}_{M}=\frac{1}{2} \int_{M}\left|\not \partial_{v} f\right|^{2} \operatorname{dvol}_{M}+\int_{M}\left\langle f, \not \partial_{v} f\right\rangle \operatorname{dvol}_{M} .
$$

(See (28) and (29) below.) Hence every solution $f: M \rightarrow \mathbb{H}$ of the Fueter equation is constant, so $v$ is a normal regular divergence-free frame.

Example 3.3 Consider the 3-sphere $M=S^{3} \subset \mathbb{H}$. The frame

$$
v_{1}(y)=2^{2 / 3} \mathbf{i} y, \quad v_{2}(y)=-2^{-1 / 3} \mathbf{j} y, \quad v_{3}(y)=-2^{-1 / 3} \mathbf{k} y,
$$

is a normal singular divergence-free frame on the 3-sphere for the standard volume form. The obvious inclusion $f: S^{3} \rightarrow \mathbb{H}$ is a nonconstant solution of the Fueter equation.

This example shows that $\mathcal{V}^{\text {sing }} \cap \mathcal{V}^{\prime} \neq \emptyset$, where $\mathcal{V}^{\prime} \subset \mathcal{V}$ denotes the connected component of the standard frame on $S^{3}$. Since $\pi_{1}\left(\mathcal{V}^{\prime}\right) \cong \mathbb{Z}_{2} \times \mathbb{Z}_{2}$ is a finite group (see Lemma 2.7(iii)) it follows that $\mathcal{V}^{\text {reg }} \cap \mathcal{V}^{\prime}$ is disconnected (see Remark 2.6).

Example 3.4 Consider the product $M:=S^{1} \times S^{2}$, where $S^{1}$ is the unit circle with coordinate $e^{\mathbf{i} \theta}$ and $S^{2} \subset \mathbb{R}^{3}$ is the unit sphere with coordinates $y_{1}, y_{2}, y_{3}$. The standard volume form is $\operatorname{dvol}_{M}:=d \theta \wedge \operatorname{dvol}_{S^{2}}$, where $\mathrm{dvol}_{S^{2}}:=y_{1} d y_{2} d y_{3}+y_{2} d y_{3} d y_{1}+y_{3} d y_{1} d y_{2}$. Define $v_{i}, w_{i}, \alpha_{i}, \beta_{i}$ by

$$
\begin{aligned}
v_{1}:=y_{1} \frac{\partial}{\partial \theta}+y_{2} \frac{\partial}{\partial y_{3}}-y_{3} \frac{\partial}{\partial y_{2}}, & \alpha_{1}:=y_{1} d \theta+y_{2} d y_{3}-y_{3} d y_{2}, \\
w_{1}:=2 y_{1} \frac{\partial}{\partial \theta}+y_{2} \frac{\partial}{\partial y_{3}}-y_{3} \frac{\partial}{\partial y_{2}}, & \beta_{1}:=y_{1} d \theta+\frac{1}{2}\left(y_{2} d y_{3}-y_{3} d y_{2}\right),
\end{aligned}
$$

for $i=1$ and by cyclic permutation for $i=2,3$. Then $v_{1}, v_{2}, v_{3}$ is a divergence-free orthonormal frame and $\alpha_{1}, \alpha_{2}, \alpha_{3}$ is the dual frame. Moreover,

$$
w_{i}=\left[v_{j}, v_{k}\right], \quad d \beta_{i}=\alpha_{j} \wedge \alpha_{k}=\iota\left(v_{i}\right) \operatorname{dvol}_{M}
$$

for every cyclic permutation $i, j, k$ of $1,2,3$.

The energy identity (20) takes the form

$$
\frac{1}{2} \int_{M}|d f|^{2} \operatorname{dvol}_{M}=\frac{1}{2} \int_{M}\left|\not \partial_{v} f\right|^{2} \operatorname{dvol}_{M}+\frac{1}{2} \int_{M}\left\langle f, \not \partial_{v} f\right\rangle \operatorname{dvol}_{M}+\widehat{\mathcal{A}}(f),
$$


where

$$
\widehat{\mathcal{A}}(f):=-\sum_{i} \int_{M} \widehat{\alpha}_{i} \wedge f^{*} \omega_{i}, \quad \widehat{\alpha}_{i}:=\frac{1}{2}\left(y_{j} d y_{k}-y_{k} d y_{j}\right),
$$

for every cyclic permutation $i, j, k$ of $1,2,3$. For $y \in S^{2}$ define

$$
\omega_{y}:=y_{1} \omega_{1}+y_{2} \omega_{2}+y_{3} \omega_{3} \in \Omega^{2}(\mathbb{H}) .
$$

Then

$$
\widehat{\mathcal{A}}(f)=\frac{1}{2} \int_{S^{2}} \int_{0}^{2 \pi} \omega_{y}\left(\partial_{\theta} f, f\right) d \theta \operatorname{dvol}_{S^{2}} .
$$

(This discussion extends to maps with values in any hyperkähler manifold $X$, if the second summand in (21) is replaced by $\mathcal{A}(f):=-\sum_{i} \int_{M} \beta_{i} \wedge f^{*} \omega_{i}$ and the integrand in (22) by the symplectic action of the loop $\theta \mapsto f\left(e^{\mathrm{i} \theta}, y\right)$ with respect to $\omega_{y}$.)

The isoperimetric inequality asserts that

$$
\frac{1}{2} \int_{0}^{2 \pi} \omega_{y}\left(\partial_{\theta} f, f\right) d \theta \leq \frac{1}{2} \int_{0}^{2 \pi}\left|\partial_{\theta} f\right|^{2} d \theta, \quad y \in S^{2} .
$$

This inequality is sharp (see [27, Sect. 4.4]). Now let $f: S^{1} \times S^{2} \rightarrow \mathbb{H}$ be a solution of the Fueter equation. By (21), (22), and (23) it satisfies

$$
\frac{1}{2} \int_{M}|d f|^{2} \mathrm{dvol}_{M}=\widehat{\mathcal{A}}(f) \leq \frac{1}{2} \int_{M}\left|\partial_{\theta} f\right|^{2} \operatorname{dvol}_{M} .
$$

Since

$$
\frac{\partial}{\partial \theta}=\sum_{i} y_{i} v_{i}
$$

is a unit vector field, equality must hold in (24) and

$$
|d f| \equiv\left|\partial_{\theta} f\right|
$$

Hence $f$ is independent of the variable in $S^{2}$. Thus the derivative of $f$ has rank one everywhere and so $f$ is constant. This shows that $v_{1}, v_{2}, v_{3}$ is a normal regular divergence-free frame.

\section{Hyperkähler Floer theory}

Fix a closed connected oriented three-manifold $M$, a volume form $\operatorname{dvol}_{M}$, a divergencefree frame $v \in \mathcal{V}\left(M, \mathrm{dvol}_{M}\right)$, and a closed hyperkähler manifold $X$ with symplectic forms $\omega_{1}, \omega_{2}, \omega_{3}$ and complex structures $J_{1}, J_{2}, J_{3}$. Denote the hyperkähler metric on $X$ by $\langle\cdot, \cdot\rangle=$ $\omega_{i}\left(\cdot, J_{i} \cdot\right)$. Consider the Fueter equation with a Hamiltonian perturbation. It has the form

$$
J_{1} \partial_{v_{1}} f+J_{2} \partial_{v_{2}} f+J_{3} \partial_{v_{3}} f=\nabla H(f)
$$

for a map $f: M \rightarrow X$. The left hand side of (25) will still be denoted by $\not \partial_{v} f$. The operator $\not_{v}$ should now be thought of as a vector field on the infinite-dimensional space $\mathcal{F}:=C^{\infty}(M, X)$ of all smooth maps from $M$ to $X$. The perturbation is determined by a 
smooth function $H: M \times X \rightarrow \mathbb{R}$ and $\nabla H$ is the gradient with respect to the variable in $X$. A solution $f$ of (25) is called nondegenerate if the linearized operator of (25) is bijective. The next theorem is a hyperkähler analogue of the Arnold conjecture for Hamiltonian systems on the torus as proved by Conley-Zehnder [4].

Theorem 4.1 [19, 20, 23, 24] Assume $v \in \mathcal{V}^{\text {reg }}$ and $X$ is flat. If every contractible solution of (25) is nondegenerate then their number is bounded below by $\operatorname{dim} H^{*}\left(X ; \mathbb{Z}_{2}\right)$. In particular, (25) has a contractible solution for every $H$.

There are two proofs of Theorem 4.1. One is due to Sonja Hohloch, Gregor Noetzel, and the present author. It is based on a hyperkähler analogue of Floer theory and is carried out in $[23,24]$ for the 3-sphere and the 3-torus. The second proof is due to Viktor Ginzburg and Doris Hein, is based on finite-dimensional reduction, and is carried out in full generality in $[19,20]$. Both proofs rely on the following fundamental estimate.

Lemma 4.2 Assume $v \in \mathcal{V}^{\text {reg }}$ and $X$ is flat. Then there is a constant $c$ such that every contractible smooth map $f: M \rightarrow X$ satisfies

$$
\int_{M}|d f|^{2} \mathrm{dvol}_{M} \leq c \int_{M}\left|\not \partial_{v} f\right|^{2} \operatorname{dvol}_{M}
$$

Proof Since $X$ is flat, it is isomorphic to a quotient of a torus $\mathbb{H}^{n} / \Lambda$ by the free action of a finite group. Hence every contractible map $f: M \rightarrow X$ lifts to a map from $M$ to $\mathbb{H}^{n}$. Thus it suffices to prove (26) for $f: M \rightarrow \mathbb{H}$. By Lemma 2.1 and Lemma B.1, the operator $\partial_{v}: W_{0}^{1,2}(M, \mathbb{H}) \rightarrow L_{0}^{2}(M, \mathbb{H})$ is Fredholm and has index zero. Since $v \in \mathcal{V}^{\text {reg }}$, this operator is bijective. Hence, for functions $f: M \rightarrow \mathbb{H}$ with mean value zero, (26) follows from the inverse operator theorem. Adding a constant to $f$ does not affect (26).

Here is an outline of the Floer theory proof of Theorem 4.1. The space $\mathcal{F}$ of maps from $M$ to $X$ carries a natural 1 -form $\Psi_{f}: T_{f} \mathcal{F} \rightarrow \mathbb{R}$ defined by

$$
\Psi_{f}(\hat{f})=\sum_{i} \int_{M} \omega_{i}\left(\partial_{v_{i}} f, \hat{f}\right) \operatorname{dvol}_{M}
$$

for a vector field $\hat{f} \in T_{f} \mathcal{F}=\Omega^{0}\left(M, f^{*} T X\right)$ along $f$. This 1 -form is closed because the vector fields $v_{i}$ are divergence-free.

Remark 4.3 Assume that the 2-forms $\iota\left(v_{i}\right) \mathrm{dvol}_{M}$ are exact and choose 1-forms $\beta_{i} \in \Omega^{1}(M)$ such that $d \beta_{i}=\iota\left(v_{i}\right) \mathrm{dvol}_{M}$ for $i=1,2,3$. Then the 1 -form $\Psi$ in (27) is the differential of the hyperkähler action functional

$$
\mathcal{A}(f):=-\sum_{i} \int_{M} \beta_{i} \wedge f^{*} \omega_{i}, \quad f \in \mathcal{F} .
$$

Remark 4.4 Assume $X$ is flat and let $\mathcal{F}_{0} \subset \mathcal{F}$ be the connected component of the constant maps. Then the restriction of $\Psi$ to $\mathcal{F}_{0}$ is the differential of the action functional $\mathcal{A}=\mathcal{A}_{v}$ : $\mathcal{F}_{0} \rightarrow \mathbb{R}$, defined by

$$
\mathcal{A}(f):=\frac{1}{2} \int_{M}\left\langle f, \not_{v} f\right\rangle \operatorname{dvol}_{M}, \quad f \in \mathcal{F}_{0} .
$$


To understand the right hand side, lift $f$ to a function with values in $\mathbb{H}^{n}$ and observe that the integrand is invariant under the action of the hyperkähler isometry group of $\mathbb{H}^{n}$.

Assume from now on that $X$ is flat. The contractible solutions of (25) are the critical points of the perturbed hyperkähler action functional $\mathcal{A}_{H}=\mathcal{A}_{v, H}: \mathcal{F}_{0} \rightarrow \mathbb{R}$, defined by

$$
\mathcal{A}_{H}(f):=\mathcal{A}(f)-\int_{M} H(f) \operatorname{dvol}_{M}, \quad f \in \mathcal{F}_{0} .
$$

The gradient flow lines of $\mathcal{A}_{H}$ are the solutions $u: \mathbb{R} \times M \rightarrow X$ of the perturbed fourdimensional Fueter equation

$$
\begin{aligned}
& \partial_{s} u+J_{1} \partial_{v_{1}} u+J_{2} \partial_{v_{2}} u+J_{3} \partial_{v_{3}} u=\nabla H(u), \\
& \lim _{s \rightarrow \infty} u(s, y)=f^{ \pm}(y) .
\end{aligned}
$$

Here $f^{ \pm} \in \mathcal{F}_{0}$ are solutions of (25). The convergence in (31) is in the $C^{\infty}$ topology on $M$ and exponential in $s$. The solutions of (30) and (31) satisfy the usual energy identity

$$
\mathcal{E}(u):=\int_{-\infty}^{\infty} \int_{M}\left|\partial_{s} u\right|^{2} \operatorname{dvol}_{M} d s=\mathcal{A}_{H}\left(f^{-}\right)-\mathcal{A}_{H}\left(f^{+}\right) .
$$

By Lemma 4.2, the energy controls the $W^{1,2}$-norms of the solutions of (30) on every compact subset of $\mathbb{R} \times M$. Since the leading term in (30) is a linear elliptic operator, this suffices for the standard regularity and compactness arguments in symplectic Floer theory to extend to the present setting. For $M=S^{3}$ the proof is carried out in detail in [24, Sect. 3] and the arguments extend verbatim to general three-manifolds. The same holds for unique continuation and transversality in [24, Sect. 4]. An index formula involving the spectral flow shows that there is a function $\mu_{H}: \operatorname{Crit}\left(\mathcal{A}_{H}\right) \rightarrow \mathbb{Z}$ such that the Fredholm index of the linearized operator of equation (30) is equal to the difference $\mu_{H}\left(f^{-}\right)-\mu_{H}\left(f^{+}\right)$for every solution $u$ of (30) and (31) (see [24, Sect. 4]). The third ingredient in the analysis is a gluing result and it follows from a standard adaptation of Floer's gluing theorem [11-13] to the hyperkähler setting. The upshot is, that the contractible solutions of (25) generate a Floer chain complex

$$
\mathrm{CF}_{*}^{\mathrm{hk}}\left(M, X, \tau_{0} ; v, H\right):=\bigoplus_{f \in \operatorname{Crit}\left(\mathcal{A}_{H}\right)} \mathbb{Z}_{2} f
$$

with $\mathbb{Z}_{2}$ coefficients. Here $\tau_{0} \in \pi_{0}(\mathcal{F})$ denotes the homotopy class of the constant maps. The Floer complex is graded by the index function $\mu_{H}$, and the boundary operator $\partial$ : $\mathrm{CF}_{k}^{\mathrm{hk}}\left(M, X, \tau_{0} ; v, H\right) \rightarrow \mathrm{CF}_{k-1}^{\mathrm{hk}}\left(M, X, \tau_{0} ; v, H\right)$ is defined by the mod two count of the solutions of (30) and (31) (modulo time shift) in the case $\mu_{H}\left(f^{-}\right)-\mu_{H}\left(f^{+}\right)=1$. The

\section{hyperkähler Floer homology groups}

$$
\mathrm{HF}_{*}^{\mathrm{hk}}\left(M, X, \tau_{0} ; v, H\right):=\operatorname{ker} \partial / \mathrm{im} \partial
$$

are independent of the regular Hamiltonian perturbation up to canonical isomorphism. Theorem 4.1 then follows from the fact that $\mathrm{HF}_{*}^{\mathrm{hk}}\left(M, X, \tau_{0} ; v, H\right)$ is isomorphic to $H_{*}\left(X ; \mathbb{Z}_{2}\right)$. For the standard divergence-free frame on the 3-sphere this was proved in [24, Sect. 5] and the argument again carries over verbatim to the general setting. 
Remark 4.5 Assume $v \in \mathcal{V}^{\text {reg }}$, let $X=\mathbb{H}^{n} / \Lambda$ be a hyperkähler torus, and let $\mathcal{F}_{\tau} \subset \mathcal{F}$ be a connected component of $\mathcal{F}$. Then the unperturbed Fueter equation $\not_{v} f=0$ may have a nonconstant solution $f_{0} \in \mathcal{F}_{\tau}$. (Examples are discussed in $[23,24]$.) In this case (26) cannot hold for $f \in \mathcal{F}_{\tau}$. However, $X$ is an additive group and, for $f \in \mathcal{F}_{\tau}$, the difference $f-f_{0}$ lifts to a function with values in the universal cover $\mathbb{H}^{n}$. Hence it follows from Lemma 4.2 that there is a constant $c_{\tau}>0$ such that every $f \in \mathcal{F}_{\tau}$ satisfies the inequality

$$
\int_{M}|d f|^{2} \operatorname{dvol}_{M} \leq c_{\tau} \int_{M}\left(\left|\not \partial_{v} f\right|^{2}+1\right) \operatorname{dvol}_{M}
$$

Moreover, the restriction of the 1 -form $\Psi$ in (27) to $\mathcal{F}_{\tau}$ is still exact. It is the differential of the action functional $\mathcal{A}_{\tau}: \mathcal{F}_{\tau} \rightarrow \mathbb{R}$ given by

$$
\mathcal{A}_{\tau}(f):=\frac{1}{2} \int_{M}\left\langle f-f_{0}, \not \partial_{v} f\right\rangle \operatorname{dvol}_{M}, \quad f \in \mathcal{F}_{\tau}
$$

To understand this, use the fact that the tangent bundle of $X$ is trivial, lift $f-f_{0}$ to a function with values in $\mathbb{H}^{n}$, and note that the integral is independent of the choice of the lift. Thus the construction of the Floer homology groups carries over to $\mathcal{F}_{\tau}$. In favourable cases the Floer homology groups $\mathrm{HF}_{*}^{\text {hk }}(M, X, \tau ; v, H)$ can be computed with the methods of [33]. They are invariant under the action of the group of volume preserving diffeomorphism of $M$ on the set of triples $(\tau, v, H)$. In general, they will not be invariant under deformation of the divergence-free frame $v$.

Remark 4.6 It would be interesting to understand the behavior of the solutions of the Fueter equation (25) as $v$ approaches a singular frame.

Remark 4.7 Another interesting question is whether the construction of the Floer homology groups can be extended to nonflat target manifolds $X$. The key obstacle is noncompactness for the solutions to the Fueter equation. The expected phenomenon, which can be demonstrated in examples, is bubbling along codimension two submanifolds of $M$. Important progress in understanding this phenomenon was recently made by Thomas Walpuski [37]. His work will be an essential ingredient in the conjectural development of a general hyperkähler Floer theory.

\section{Relation to Donaldson-Thomas theory}

The discussion in this section is speculative. It concerns the relation between the DonaldsonThomas-Floer theory of a product manifold $Y=M \times \Sigma$ (where $\Sigma$ is a hyperkähler 4-manifold) and hyperkähler Floer theory.

Let $Y$ be a closed connected 7-manifold. A 3-form $\phi \in \Omega^{3}(Y)$ is called nondegenerate if, for any two linearly independent tangent vectors $u, v$, there is a third tangent vector $w$ such that $\phi(u, v, w) \neq 0$. Every nondegenerate 3-form $\phi$ determines a unique Riemannian metric on $Y$ such that the bilinear form $(u, v) \mapsto u \times v$ on $T Y$, defined by $\langle u \times v, w\rangle:=$ $\phi(u, v, w)$, is a cross product, i.e. it satisfies $|u \times v|^{2}=|u|^{2}|v|^{2}-\langle u, v\rangle^{2}$ for all $u, v \in T_{y} Y$ (see e.g. [35]). A nondegenerate 3-form also determines a unique orientation on $Y$ such 
that the 7-form $\iota(u) \phi \wedge \iota(u) \phi \wedge \phi$ is positive for every nonzero tangent vector $u \in T Y$. A $\mathbf{G}_{2}$-structure on $Y$ is a nondegenerate 3 -form $\phi$ that is harmonic with respect to the Riemannian metric determined by $\phi$.

Assume $\phi \in \Omega^{3}(Y)$ is a $\mathrm{G}_{2}$-structure and denote $\psi:=* \phi \in \Omega^{4}(Y)$. Fix a compact Lie group $\mathrm{G}$ and let $\mathcal{A}(Y)$ be the space of connections on a principal G-bundle over $Y$. For this discussion it suffices to think of $\mathcal{A}(Y)$ as the space of 1-forms on $Y$ with values in the Lie algebra $\mathfrak{g}=\operatorname{Lie}(\mathrm{G})$. The Donaldson-Thomas-Chern-Simons functional $\mathrm{CS}^{\psi}: \mathcal{A}(Y) \rightarrow$ $\mathbb{R}$ is defined by

$$
\operatorname{CS}^{\psi}(\mathbb{A}):=\int_{Y} \operatorname{cs}_{\mathbb{A}_{0}}(\mathbb{A}) \wedge \psi
$$

where $\mathbb{A}_{0} \in \mathcal{A}(Y)$ is a reference connection and $\operatorname{cs}_{\mathbb{A}_{0}}(\mathbb{A}) \in \Omega^{3}(Y)$ denotes the ChernSimons 3-form

$$
\operatorname{cs}_{\mathbb{A}_{0}}(\mathbb{A}):=\left\langle a \wedge\left(F_{\mathbb{A}_{0}}+\frac{1}{2} d_{\mathbb{A}_{0}} a+\frac{1}{6}[a \wedge a]\right)\right\rangle, \quad a:=\mathbb{A}-\mathbb{A}_{0} .
$$

A critical point of $\mathrm{CS}^{\psi}$ is a connection $\mathbb{A} \in \mathcal{A}(Y)$ whose curvature $F_{\mathbb{A}}$ satisfies

$$
F_{\mathbb{A}} \wedge \psi=0
$$

The solutions are $\mathbf{G}_{2}$-instantons. A negative gradient flow line of $\mathrm{CS}^{\psi}$ is (gauge equivalent to) a pair, consisting of a path $\mathbb{R} \rightarrow \mathcal{A}(Y): s \mapsto \mathbb{A}(s)$ of connections and a path $\mathbb{R} \rightarrow \Omega^{0}(Y, \mathfrak{g}): s \mapsto \Phi(s)$ of sections of the Lie algebra bundle, that satisfy

$$
\partial_{s} \mathbb{A}-d_{\mathbb{A}} \Phi+*\left(F_{\mathbb{A}} \wedge \psi\right)=0 .
$$

The basic idea of Donaldson-Thomas theory in the $\mathrm{G}_{2}$ setting is to define Floer homology groups $\mathrm{HF}_{*}^{\mathrm{DT}}(Y)$, generated by the gauge equivalence classes of solutions of (33), with the boundary operator given by counting the gauge equivalence classes of solutions of (34) (see $[6,7])$.

Now let $M$ be a closed connected oriented 3-manifold, equipped with a volume form $\operatorname{dvol}_{M}$ and a normal regular divergence-free frame $v \in \mathcal{V}^{\text {reg }}$. Denote by $\alpha_{1}, \alpha_{2}, \alpha_{3}$ the dual frame in $\Omega^{1}(M)$. Then the 2-forms $\alpha_{i} \wedge \alpha_{j}$ are closed for all $i$ and $j$ (see Remark 2.9). Let $\Sigma$ be a closed connected hyperkähler 4-manifold (i.e. either a 4-torus or a $K 3$ surface) with symplectic forms $\sigma_{1}, \sigma_{2}, \sigma_{3}$ and complex structures $j_{1}, j_{2}, j_{3}$. These structures determine a nondegenerate 3 -form $\phi$ on the product $Y:=M \times \Sigma$, given by

$$
\begin{aligned}
\phi & :=\operatorname{dvol}_{M}-\alpha_{1} \wedge \sigma_{1}-\alpha_{2} \wedge \sigma_{2}-\alpha_{3} \wedge \sigma_{3} \\
\psi & :=\operatorname{dvol}_{\Sigma}-\alpha_{2} \wedge \alpha_{3} \wedge \sigma_{1}-\alpha_{3} \wedge \alpha_{1} \wedge \sigma_{2}-\alpha_{1} \wedge \alpha_{2} \wedge \sigma_{3}
\end{aligned}
$$

Here differential forms on $M$ and $\Sigma$ are identified with their pullbacks to $Y=M \times \Sigma$. The Riemannian metric on $M \times \Sigma$ determined by $\phi$ is the product metric and the cross product is given by $v_{1} \times v_{2}=v_{3}$ and $\xi \times v_{i}=j_{i} \xi$ for $\xi \in T \Sigma$ and $i=1,2,3$.

Fix a principal G-bundle $Q \rightarrow \Sigma$ and consider the product G-bundle $M \times Q \rightarrow M \times \Sigma$. Denote by $\mathcal{A}(\Sigma)$ the space of connections on $Q$ and by $\mathfrak{g}_{Q} \rightarrow \Sigma$ the Lie algebra bundle. Write a connection on $\mathbb{R} \times M \times \Sigma$ in the form $A+\Phi d s+\sum_{i} \Psi_{i} \alpha_{i}$ with $A: \mathbb{R} \times M \rightarrow \mathcal{A}(\Sigma)$ 
and $\Phi, \Psi_{i}: \mathbb{R} \times M \rightarrow \Omega^{0}\left(\Sigma, \mathfrak{g}_{Q}\right)$. Then equation (34) has the following form (with $\varepsilon=1$ )

$$
\begin{aligned}
\partial_{s} A-d_{A} \Phi+\sum_{i}\left(\partial_{v_{i}} A-d_{A} \Psi_{i}\right) \circ j_{i} & =0, \\
& F_{01}+F_{23}-\varepsilon^{-2}\left\langle F_{A}, \sigma_{1}\right\rangle=0, \\
& F_{02}+F_{31}-\varepsilon^{-2}\left\langle F_{A}, \sigma_{2}\right\rangle=0, \\
& F_{03}+F_{12}-\varepsilon^{-2}\left\langle F_{A}, \sigma_{3}\right\rangle=0 .
\end{aligned}
$$

Here $F_{0 i}=F_{\mathbb{A}+\Phi d s}\left(\partial / \partial s, v_{i}\right)$ and $F_{j k}:=F_{\mathbb{A}+\Phi d s}\left(v_{j}, v_{k}\right)$. Thus

$$
\begin{aligned}
F_{0 i} & :=\partial_{s} \Psi-\partial_{v_{i}} \Phi+\left[\Phi, \Psi_{i}\right] \\
F_{j k} & :=\partial_{v_{j}} \Psi_{k}-\partial_{v_{k}} \Psi_{j}+\left[\Psi_{j}, \Psi_{k}\right]+\sum_{i} \alpha_{i}\left(\left[v_{j}, v_{k}\right]\right) \Psi_{i}
\end{aligned}
$$

For general $\varepsilon$ equation (36) is obtained by replacing $\sigma_{i}$ with $\varepsilon^{2} \sigma_{i}$ and $\mathrm{dvol}_{\Sigma}$ with $\varepsilon^{4} \mathrm{dvol}_{\Sigma}$. Taking the limit $\varepsilon \rightarrow 0$ in (36) one obtains the equation

$$
\partial_{S} A-d_{A} \Phi-\sum_{i}\left(\partial_{v_{i}} A-d_{A} \Psi_{i}\right) \circ j_{i}=0, \quad F_{A}^{+}=0 .
$$

This is the unperturbed Fueter equation (30) on $\mathbb{R} \times M$ with values in the moduli space $X=\mathcal{M}^{\text {asd }}(\Sigma)$ of anti-self-dual instantons on $\Sigma$ with its standard hyperkähler structure (see Remark 6.5 below). These observations suggests a correspondence between the DonaldsonThomas-Floer homology groups $\mathrm{HF}_{*}^{\mathrm{DT}}(M \times \Sigma)$ and the hyperkähler Floer homology groups $\mathrm{HF}_{*}^{\mathrm{hk}}\left(M, \mathcal{M}^{\text {asd }}(\Sigma)\right)$, in analogy to the Atiyah-Floer conjecture [8].

Remark 5.1 (i) The 4-form $\psi$ in (35) is closed when $\lambda=\operatorname{dvol}_{M}\left(v_{1}, v_{2}, v_{3}\right)$ is constant, however, the 3 -form $\phi$ will in general not be closed.

(ii) Consider the 7-manifold $Y:=M \times \Sigma$, where $\Sigma$ is a $K 3$-surface and $M$ is a closed oriented 3-manifold with $b_{1}(M) \leq 2$. Let $\pi: Y \rightarrow \Sigma$ be the projection. The following argument by Donaldson shows that $Y$ does not admit a $\mathrm{G}_{2}$-structure. On a $\mathrm{G}_{2}$-manifold there is a splitting of the space $H^{2}(Y)$ of harmonic 2-forms into the eigenspaces

$$
\begin{aligned}
& H^{2,+}(Y):=\left\{\tau \in H^{2}(Y) \mid *(\phi \wedge \tau)=2 \tau\right\}=\left\{*(\psi \wedge \alpha) \mid \alpha \in H^{1}(Y)\right\}, \\
& H^{2,-}(Y):=\left\{\tau \in H^{2}(Y) \mid *(\phi \wedge \tau)=-\tau\right\}=\left\{\tau \in H^{2}(Y) \mid \psi \wedge \tau=0\right\} .
\end{aligned}
$$

The subspace $H^{2,+}(Y)$ is isomorphic to $H^{1}(Y)$ and, by definition, the quadratic form $H^{2,-}(Y) \rightarrow \mathbb{R}: \tau \mapsto \int_{Y} \phi \wedge \tau \wedge \tau$ is negative definite. In the case at hand, $H^{2,-}(Y)$ has codimension $b_{1}(Y)=b_{1}(M) \leq 2$, and hence intersects $\pi^{*} H^{2, \pm}(\Sigma)$ nontrivially. Choose $0 \neq$ $\tau^{ \pm} \in H^{2, \pm}(\Sigma)$ such that $\pi^{*} \tau^{ \pm} \in H^{2,-}(Y)$ and $\tau^{ \pm} \wedge \tau^{ \pm}= \pm \operatorname{dvol}_{\Sigma}$. Then $\int_{Y} \phi \wedge \pi^{*}\left(\tau^{-} \wedge \tau^{-}\right)$ and $\int_{Y} \phi \wedge \pi^{*}\left(\tau^{+} \wedge \tau^{+}\right)$have opposite signs, a contradiction.

(iii) The argument in (ii) breaks down for $M=\mathbb{T}^{3}$ and in this case the 3-form $\phi$ in (35) is indeed a $\mathrm{G}_{2}$-structure (for a suitable frame on $\mathbb{T}^{3}$ ).

(iv) Existence results for $\mathrm{G}_{2}$-structures were established by Joyce [25]. A question posed by Donaldson is, which 7-manifolds admit nondegenerate 3-forms $\phi$ that are closed or coclosed, but not necessarily both. This is analogous to the question, which manifolds admit symplectic or complex structures, but not necessarily Kähler structures. 
Remark 5.2 The above setting extends naturally to general G-bundles over $M \times \Sigma$. A homotopy class of maps from $M$ to the moduli space $\mathcal{M}^{\text {asd }}(\Sigma)$ determines the relevant principal G-bundle over $M \times \Sigma$.

Remark 5.3 The discussion of the present section is closely related to several observations by Donaldson-Thomas in [6] and by Donaldson-Segal in [7]. In [6, Sect. 5] Donaldson and Thomas discuss Spin(7)-instantons on a product $S \times \Sigma$ of two hyperkähler 4-manifolds $S$ and $\Sigma$. They note that, shrinking the metric on $\Sigma$, leads in the adiabatic limit to solutions of the Fueter equation for maps $u: S \rightarrow \mathcal{M}^{\text {asd }}(\Sigma)$. Taking $S=\mathbb{R} \times M$ one arrives at equation (36).

In [7, Sect. 6] Donaldson and Segal extend this discussion to a setting where $M \times \Sigma$ is replaced by a $\mathrm{G}_{2}$-manifold $Y$, and $M$ is replaced by an associative submanifold of $Y$. This leads to an interaction between $\mathrm{G}_{2}$-instantons on $Y$ and Fueter sections of the bundle of framed anti-self-dual instantons on the fibers of the normal bundle of $M$. Generically, such Fueter sections are expected to exist at isolated parameters in a 1-parameter family of $\mathrm{G}_{2}$ structures. The existence of nonconstant solutions of the Fueter equation for singular divergence-free frames, as discussed in Sect. 2, seems to be a linear analogue of this codimension one phenomenon.

In this extended setting, relating the Donaldson-Thomas equations over a $G_{2}$-manifold $Y$ to the Fueter equations over an associative submanifold $M$, important progress has recently been made by Walpuski $[38,39]$. He carried out the adiabatic limit analysis and proved that Fueter sections over $M$, under suitable transversality assumptions, give rise to $\mathrm{G}_{2}$-instantons over $Y$ whose energy is concentrated near $M$.

\section{The gauged Fueter equation}

Equation (36) extends naturally to a setting where the space of connections over $\Sigma$ is replaced by a hyperkähler manifold $\left(X, \omega_{1}, \omega_{2}, \omega_{3}, J_{1}, J_{2}, J_{3}\right)$ and the group of gauge transformations over $\Sigma$ by a compact Lie group G, acting on $X$ by hyperkähler isometries. Denote the action by $(g, x) \mapsto g x$ and the infinitesimal action of the Lie algebra $\mathfrak{g}:=\operatorname{Lie}(\mathrm{G})$ by $L_{x}: \mathfrak{g} \rightarrow T_{x} X$. Thus $L_{x} \xi:=\left.\frac{d}{d t}\right|_{t=0} \exp (t \xi) x$ for $x \in X$ and $\xi \in \mathfrak{g}$. Choose an invariant inner product on $\mathfrak{g}$ and suppose that the action is generated by equivariant moment maps $\mu_{1}, \mu_{2}, \mu_{3}: X \rightarrow \mathfrak{g}$, so that

$$
\omega_{i}\left(L_{x} \xi, \hat{x}\right)=\left\langle d \mu_{i}(x) \hat{x}, \xi\right\rangle, \quad \omega_{i}\left(L_{x} \xi, L_{x} \eta\right)=\left\langle\mu_{i}(x),[\xi, \eta]\right\rangle,
$$

for all $\hat{x} \in T_{x} X, \xi, \eta \in \mathfrak{g}$, and $i=1,2,3$. Fix an oriented 3-manifold $M$ with a volume form $\operatorname{dvol}_{M}$, a normal divergence-free frame $v_{1}, v_{2}, v_{3}$, and denote by $\alpha_{1}, \alpha_{2}, \alpha_{3} \in \Omega^{1}(M)$ the dual frame. Choose a principal G-bundle $\pi: P \rightarrow M$, let $\mathcal{A} \subset \Omega^{1}(P, \mathfrak{g})$ be the space of connections on $P$, and let $\mathcal{F}$ be the space of G-equivariant maps $f: P \rightarrow X$. There is a natural 1-form on $\mathcal{A} \times \mathcal{F}$, which assigns to every pair $(A, f) \in \mathcal{A} \times \mathcal{F}$ the linear map $\Psi_{A, f}: T_{A} \mathcal{A} \times T_{f} \mathcal{F} \rightarrow \mathbb{R}$, given by

$$
\begin{aligned}
\Psi_{A, f}(\hat{A}, \hat{f}) & :=\int_{M}\left\langle F_{A} \wedge \hat{A}\right\rangle-\sum_{i} \int_{M}\left(\omega_{i}\left(d_{A} f\left(v_{i}\right), \hat{f}\right)+\left\langle\mu_{i}(f), \hat{A}\left(v_{i}\right)\right\rangle\right) \mathrm{dvol}_{M} \\
& =\sum_{i} \int_{M}\left\langle F_{A}\left(v_{j}, v_{k}\right)-\mu_{i}(f), \hat{A}\left(v_{i}\right)\right\rangle \operatorname{dvol}_{M}-\int_{M}\left\langle\sum_{i} J_{i} d_{A} f\left(v_{i}\right), \hat{f}\right\rangle \mathrm{dvol}_{M}
\end{aligned}
$$


for $\hat{A} \in T_{A} \mathcal{A}=\Omega^{1}\left(M, \mathfrak{g}_{P}\right)$ and $\hat{f} \in T_{f} \mathcal{F}=\Omega^{0}\left(M, f^{*} T X / G\right)$. Here the second sum runs over all cyclic permutations $i, j, k$ of $1,2,3$. The 1 -form $d_{A} f: T P \rightarrow f^{*} T X$ is the covariant derivative of $f$ with respect to $A$, defined by $\left(d_{A} f\right)_{p}(\hat{p}):=d f(p) \hat{p}+L_{f(p)} A_{p}(\hat{p})$ for $\hat{p} \in$ $T_{p} P$. It is G-equivariant and horizontal (i.e. $\left(d_{A} f\right)_{p}(p \xi)=0$ for $\left.\xi \in \mathfrak{g}\right)$. Hence it descends to a 1 -form on $M$ with values in the quotient bundle $f^{*} T X / \mathrm{G} \rightarrow M$. To understand the term $d_{A} f\left(v_{i}\right) \in \Omega^{0}\left(M, f^{*} T X / \mathrm{G}\right)$, choose G-equivariant lifts $\tilde{v}_{i} \in \operatorname{Vect}(P)$ of $v_{i}$ and observe that the section $d_{A} f\left(\tilde{v}_{i}\right)$ of the vector bundle $f^{*} T X \rightarrow P$ is G-equivariant and independent of the choice of the lifts. The resulting section of the bundle $f^{*} T X / \mathrm{G} \rightarrow P / \mathrm{G}=M$ is denoted $d_{A} f\left(v_{i}\right)$.

The group $\mathcal{G}=\mathcal{G}(P)$ of gauge transformations acts contravariantly on $\mathcal{A} \times \mathcal{F}$ by $g^{*} A:=$ $g^{-1} d g+g^{-1} A g$ and $g^{*} f:=g^{-1} f$. The covariant infinitesimal action of the Lie algebra $\Omega^{0}\left(M, \mathfrak{g}_{P}\right)=\operatorname{Lie}(\mathcal{G})$ is given by $\Phi \mapsto\left(-d_{A} \Phi, L_{f} \Phi\right) \in T_{A} \mathcal{A} \times T_{f} \mathcal{F}$. The 1-form (39) is $\mathcal{G}$-invariant and horizontal, in the sense that $\Psi_{A, f}\left(-d_{A} \Phi, L_{f} \Phi\right)=0$ for all $A, f$, and $\Phi$. Hence $\Psi$ descends to a 1 -form on the quotient space $\mathcal{B}:=(\mathcal{A} \times \mathcal{F}) / \mathcal{G}$.

Remark 6.1 The 1-form (39) is closed. To see this, choose a smooth path $I \rightarrow \mathcal{A} \times \mathcal{F}: s \mapsto$ $\left(A_{s}, u_{s}\right)$. Think of $\mathbb{A}:=\left\{A_{s}\right\}_{s \in I}$ as a connection on the principal bundle $I \times P$ over $I \times M$, and of $u$ as a G-equivariant map from $I \times P$ to $X$. The integral of $\Psi$ over this path is given by

$$
\begin{aligned}
\int_{I}(\mathbb{A}, u)^{*} \Psi & =\int_{I} \Psi_{A_{s}, f_{s}}\left(\partial_{s} A, \partial_{s} u\right) d s \\
& =\int_{I \times M}\left(\frac{1}{2}\left\langle F_{\mathbb{A}} \wedge F_{\mathbb{A}}\right\rangle-\sum_{i}\left(u^{*} \omega_{i}-d\left\langle\mu_{i}(u), \mathbb{A}\right\rangle\right) \wedge \iota\left(v_{i}\right) \operatorname{dvol}_{M}\right) .
\end{aligned}
$$

The last integral is meaningful, because the 2-form $u^{*} \omega_{i}-d\left\langle\mu_{i}(u), \mathbb{A}\right\rangle$ on $I \times P$ descends to $I \times M$. Since the integrand is closed, the integral is invariant under homotopy with fixed endpoints. If $\iota\left(v_{i}\right) \mathrm{dvol}_{M}=d \beta_{i}$ and CS $: \mathcal{A} \rightarrow \mathbb{R}$ denotes the Chern-Simons functional, then (39) is the differential of the action functional

$$
\mathcal{A}(A, f):=\operatorname{CS}(A)+\sum_{i} \int_{M} \beta_{i} \wedge\left(f^{*} \omega_{i}-d\left\langle\mu_{i}(f), A\right\rangle\right) .
$$

Remark 6.2 Given $A \in \mathcal{A}$, define the twisted Fueter operator by

$$
\not \partial_{A, v} f:=J_{1} d_{A} f\left(v_{1}\right)+J_{2} d_{A} f\left(v_{2}\right)+J_{3} d_{A} f\left(v_{3}\right)
$$

for $f \in \mathcal{F}$. Thus $\not_{A, v} f$ is a section of the quotient bundle $f^{*} T X / \mathrm{G} \rightarrow M$. Then the zeros of the 1 -form (39) are the solutions $(A, f) \in \mathcal{A} \times \mathcal{F}$ of the three-dimensional gauged Fueter equation

$$
\not \partial_{A, v} f=0, \quad * F_{A}=\sum_{i}\left(\mu_{i} \circ f\right) \pi^{*} \alpha_{i} .
$$

Here $*$ denotes the Hodge $*$-operator on $M$ associated to the metric (19). Thus

$$
* F_{A}=\sum_{i} F_{A}\left(v_{j}, v_{k}\right) \pi^{*} \alpha_{i},
$$

where the sum runs over all cyclic permutations $i, j, k$ of $1,2,3$. 
The gradient flow lines are pairs, consisting of a connection $\mathbb{A}=A+\Phi d s$ on $\mathbb{R} \times P$ and a G-equivariant map $u: \mathbb{R} \times P \rightarrow X$, that satisfy the four-dimensional gauged Fueter equation

$$
\partial_{s} u+L_{u} \Phi-\not \partial_{A, v} u=0, \quad \partial_{s} A-d_{A} \Phi+* F_{A}=\sum_{i}\left(\mu_{i} \circ u\right) \pi^{*} \alpha_{i}
$$

This is reminiscent of the symplectic vortex equations [2, 3, 15, 18, 28-30]. Similar equations were studied by Taubes [36], Pidstrigatch [31], and Haydys [22]. The usual Fueter equation corresponds to the case $\mathrm{G}=\{1\}$, the instanton Floer equation [14] to the case $X=\{\mathrm{pt}\}$, the Donaldson-Thomas equation to the case $X=\mathcal{A}(\Sigma)$ and $\mathrm{G}=\mathcal{G}(\Sigma)$ (see Remark 6.5), and the Seiberg-Witten equation to the case $X=\mathbb{H}$ and $\mathrm{G}=S^{1}$ (see Sect. 7).

Remark 6.3 Define the energy of a pair $(A, f) \in \mathcal{A} \times \mathcal{F}$ by

$$
\mathcal{E}(A, f):=\frac{1}{2} \int_{M}\left(\left|d_{A} f\right|^{2}+\left|F_{A}\right|^{2}+\sum_{i}\left|\mu_{i}(f)\right|^{2}\right) \mathrm{dvol}_{M} .
$$

Then there is an energy identity

$$
\begin{array}{r}
\frac{1}{2} \int_{M}\left(\left|\not \partial_{A, v} f\right|^{2}+\left|* F_{A}-\sum_{i} \mu_{i}(f) \pi^{*} \alpha_{i}\right|^{2}\right) \mathrm{dvol}_{M} \\
=\mathcal{E}(A, f)+\sum_{i} \int_{M} \alpha_{i} \wedge\left(f^{*} \omega_{i}-d\left\langle\mu_{i}(f), A\right\rangle\right) .
\end{array}
$$

This is the gauged analogue of equation (20).

Remark 6.4 One can introduce an $\varepsilon$-parameter in (42) as follows

$$
\partial_{s} u+L_{u} \Phi-\not \partial_{A, v} u=0, \quad \partial_{s} A-d_{A} \Phi+* F_{A}=\varepsilon^{-2} \sum_{i}\left(\mu_{i} \circ u\right) \pi^{*} \alpha_{i}
$$

In the limit $\varepsilon \rightarrow 0$ one obtains, formally, the equation

$$
\partial_{s} u+L_{u} \Phi-\not \partial_{A, v} u=0, \quad \mu_{1} \circ u=\mu_{2} \circ u=\mu_{3} \circ u=0 .
$$

This corresponds to the four-dimensional Fueter equation on $\mathbb{R} \times M$ with values in the hyperkähler quotient

$$
X / / \mathrm{G}:=\left(\mu_{1}^{-1}(0) \cap \mu_{2}^{-1}(0) \cap \mu_{3}^{-1}(0)\right) / \mathrm{G} .
$$

Remark 6.5 The space $\mathcal{X}:=\mathcal{A}(\Sigma)$ of connections on a principal G-bundle $Q$ over a closed hyperkähler 4-manifold $\left(\Sigma, \sigma_{1}, \sigma_{2}, \sigma_{3}, j_{1}, j_{2}, j_{3}\right)$ is itself a hyperkähler manifold. The symplectic forms $\omega_{i} \in \Omega^{2}(\mathcal{X})$ and the complex structures $J_{i}: T \mathcal{X} \rightarrow T \mathcal{X}$ are given by

$$
\omega_{i}(\alpha, \beta):=\int_{\Sigma}\langle\alpha \wedge \beta\rangle \wedge \sigma_{i}, \quad J_{i} \alpha:=*_{\Sigma}\left(\alpha \wedge \sigma_{i}\right)=-\alpha \circ j_{i}
$$


for $\alpha, \beta \in \Omega^{1}\left(\Sigma, \mathfrak{g}_{Q}\right)=T_{A} \mathcal{A}(\Sigma)$ and $i=1,2,3$. The group $\mathcal{G}=\mathcal{G}(\Sigma)$ of gauge transformations of $Q$ acts on $\mathcal{X}=\mathcal{A}(\Sigma)$ by hyperkähler isometries and the moment maps $\mu_{i}: \mathcal{A}(\Sigma) \rightarrow \Omega^{0}\left(\Sigma, \mathfrak{g}_{Q}\right)=\operatorname{Lie}(\mathcal{G}(\Sigma))$ are

$$
\mu_{i}(A):=*_{\Sigma}\left(F_{A} \wedge \sigma_{i}\right)=\left\langle F_{A}, \sigma_{i}\right\rangle, \quad A \in \mathcal{A}(\Sigma) .
$$

If $\mathcal{P}=M \times \mathcal{G}(\Sigma)$ is the trivial bundle with the infinite-dimensional structure group $\mathcal{G}(\Sigma)$ then (45) is equivalent to the Donaldson-Thomas equation (36) on $M \times \Sigma$. The function $A: \mathbb{R} \times M \rightarrow \mathcal{A}(\Sigma)$ in (36) corresponds to $u$ in (45), while the functions $\Psi_{i}$ : $\mathbb{R} \times M \rightarrow \Omega^{0}\left(\Sigma, \mathfrak{g}_{Q}\right)$ in (36) determine the path of connections $A(s):=\sum_{i} \Psi_{i}(s) \alpha_{i} \in$ $\Omega^{1}\left(M, \Omega^{0}\left(\Sigma, \mathfrak{g}_{Q}\right)\right)$ in (45). The hyperkähler quotient is the moduli space $\mathcal{A}(\Sigma) / / \mathcal{G}(\Sigma)=$ $\mathcal{M}^{\text {asd }}(\Sigma)$ of anti-self-dual instantons on $\Sigma$. These observations extend to nontrivial $\mathcal{G}(\Sigma)$ bundles $\mathcal{P} \rightarrow M$, arising from general G-bundles over $M \times \Sigma$.

\section{Relation to Seiberg-Witten theory}

Let $M$ be a closed connected oriented Riemannian 3-manifold. A $\operatorname{spin}^{c}$ structure on $M$ is a rank two complex hermitian vector bundle $W \rightarrow M$ equipped with a Clifford action $\gamma: T M \rightarrow \operatorname{End}(W)$. This action assigns to every tangent vector $v \in T_{y} M$ a traceless endomorphism $\gamma(v)$ of the fiber $W_{y}$ satisfying

$$
\gamma(v)+\gamma(v)^{*}=0, \quad \gamma(v)^{*} \gamma(v)=|v|^{2} \mathbb{1},
$$

and it satisfies $\gamma\left(v_{3}\right) \gamma\left(v_{2}\right) \gamma\left(v_{1}\right)=\mathbb{1}$ for every positive orthonormal frame. A spin ${ }^{c}$ connection is a hermitian connection on $W$ that satisfies the Leibniz rule for Clifford multiplication (with the Levi-Civita connection on the tangent bundle). Associated to a $\operatorname{spin}^{c}$ connection $\nabla_{A}$ is a Dirac operator $\not_{A}: \Omega^{0}(M, W) \rightarrow \Omega^{0}(M, W)$ defined by

$$
\not D_{A} f:=\sum_{i} \gamma\left(v_{i}\right) \nabla_{A, v_{i}} f
$$

for $f \in \Omega^{0}(M, W)$. Here $v_{1}, v_{2}, v_{3}$ is any global orthonormal frame of $T M$. The Dirac operator is self-adjoint and independent of the choice of the frame. The difference of two $\operatorname{spin}^{c}$ connections is an imaginary valued 1-form. Let $\mathcal{A}(\gamma)$ denote the space of $\operatorname{spin}^{c}$ connections on $W$. The perturbed Chern-Simons-Dirac functional $\operatorname{CSD}_{\eta}: \mathcal{A}(\gamma) \times \Omega^{0}(M, W)$ has the form

$$
\operatorname{CSD}_{\eta}(A, f):=\int_{Y}\left\langle\left(A-A_{0}\right) \wedge\left(\eta+\frac{1}{2}\left(F_{A_{0}}+F_{A}\right)\right)\right\rangle-\frac{1}{2} \int_{Y}\left\langle f, \not D_{A} f\right\rangle \operatorname{dvol}_{M} .
$$

Here $A_{0} \in \mathcal{A}(\gamma)$ is a reference connection, $\eta \in \Omega^{2}(M, \mathbf{i} \mathbb{R})$ is a closed 2-form, and $F_{A}:=\frac{1}{2} \operatorname{trace}\left(F^{\nabla_{A}}\right) \in \Omega^{2}(M, \mathbf{i} \mathbb{R})$. A negative gradient flow line of $\mathrm{CSD}_{\eta}$ is a triple $(A, \Phi, u)$, consisting of a smooth path $\mathbb{R} \rightarrow \mathcal{A}(\gamma): s \mapsto A(s)$ of $\operatorname{spin}^{c}$ connections, a smooth path $\mathbb{R} \rightarrow \Omega^{0}(M, \mathbf{i} \mathbb{R}): s \mapsto \Phi(s)$ of functions on $M$, and a smooth path $\mathbb{R} \rightarrow \Omega^{0}(M, W): s \mapsto u_{s}=u(s, \cdot)$ of sections of $W$, that satisfy the 4-dimensional SeibergWitten-Floer equation

$$
\partial_{s} u+\Phi u-\not D_{A} u=0, \quad \partial_{s} A-d \Phi+*\left(F_{A}+\eta\right)=\gamma^{-1}\left(\left(u u^{*}\right)_{0}\right) .
$$


Here $\left(u u^{*}\right)_{0}: W \rightarrow W$ denotes the traceless hermitian endomorphism given by $\left(u u^{*}\right)_{0} w:=$ $\langle u, w\rangle u-\frac{1}{2}|u|^{2} w, I$ denotes the complex structure on $W,\langle\cdot, \cdot\rangle$ denotes the real inner product on $W$, and $\gamma^{-1}\left(\left(u u^{*}\right)_{0}\right):=\frac{\mathbf{i}}{2}\langle I \gamma(\cdot) u, u\rangle$. (See the book by Kronheimer-Mrowka [26] for a detailed account of Seiberg-Witten-Floer theory and its applications to low-dimensional topology.)

Now let $v_{1}, v_{2}, v_{3} \in \operatorname{Vect}(M)$ be an orthonormal divergence-free frame and let $\alpha_{1}, \alpha_{2}, \alpha_{3} \in$ $\Omega^{1}(M)$ be the dual frame. For an orthonormal frame the divergence-free condition can be expressed in the form

$$
\nabla_{v_{1}} v_{1}+\nabla_{v_{2}} v_{2}+\nabla_{v_{3}} v_{3}=0
$$

The frame induces a spin structure on the trivial bundle $W:=M \times \mathbb{H}$ via

$$
\gamma\left(v_{i}\right)=J_{i}, \quad i=1,2,3,
$$

where $J_{1}, J_{2}, J_{3}$ are the complex structures in (4). (This is a spin structure because it commutes with all three complex structures $I_{1}, I_{2}, I_{3}$ in (4).) The spin connection $A_{0} \in \mathcal{A}(\gamma)$ of this structure is given by

$$
\nabla_{A_{0}, v_{i}} f=\partial_{v_{i}} f+A_{0}\left(v_{i}\right) f
$$

for $f \in \Omega^{0}(M, \mathbb{H})$ and $i=1,2,3$, where

$$
A_{0}\left(v_{i}\right):=\frac{1}{2}\left\langle\nabla_{v_{i}} v_{j}, v_{k}\right\rangle J_{i}+\frac{1}{2}\left\langle\nabla_{v_{i}} v_{i}, v_{j}\right\rangle J_{k}-\frac{1}{2}\left\langle\nabla_{v_{i}} v_{i}, v_{k}\right\rangle J_{j}
$$

for every cyclic permutation $i, j, k$ of $1,2,3$. The curvature of $A_{0}$ is traceless. A simple calculation, using (48) and the orthonormal condition, shows that

$$
\not A_{A_{0}} f=\not_{v} f+\lambda f, \quad \lambda:=\frac{1}{4} \sum_{i} \alpha_{i}\left(\left[v_{j}, v_{k}\right]\right),
$$

where the sum runs over all cyclic permutations $i, j, k$ of $1,2,3$. Now consider the circle action on $\mathbb{H}$ generated by the vector field $x \mapsto-x \mathbf{i}$. This is the standard circle action associated to the complex structure $I_{1}$ in (4). It preserves the hyperkähler structure determined by the complex structures $J_{1}, J_{2}, J_{3}$ and the symplectic forms $\omega_{1}, \omega_{2}, \omega_{3}$ in (20). The moment maps $\mu_{1}, \mu_{2}, \mu_{3}: \mathbb{H} \rightarrow \mathbf{i} \mathbb{R}$ of this action are given by $\mu_{i}(x)=\frac{\mathbf{i}}{2} \omega_{i}(-x \mathbf{i}, x)$. Hence

$$
\gamma^{-1}\left(\left(u u^{*}\right)_{0}\right)=\frac{\mathbf{i}}{2}\langle-\gamma(\cdot) u \mathbf{i}, u\rangle=\sum_{i} \frac{\mathbf{i}}{2} \omega_{i}(-u \mathbf{i}, u) \alpha_{i}=\sum_{i}\left(\mu_{i} \circ u\right) \alpha_{i} .
$$

By (49) and (50), the Seiberg-Witten-Floer equation (47) has the form

$$
\partial_{s} u-u \Phi-\not \partial_{A, v} u=\lambda u, \quad \partial_{s} A-d \Phi+*(d A+\eta)=\sum_{i}\left(\mu_{i} \circ u\right) \alpha_{i} .
$$

Here $\mathbb{R} \rightarrow \Omega^{1}(M, \mathbf{i} \mathbb{R}): s \mapsto A(s)$ is a path of imaginary valued 1-forms and the associated path of $\operatorname{spin}^{c}$ connections is $s \mapsto A_{0}+A(s)$. With $\eta=0$ and $\lambda=0$ this is the gauged Fueter equation (42) for $X=\mathbb{H}$ and $\mathrm{G}=S^{1}$. This correspondence extends to general $\operatorname{spin}^{c}$ structures via the appropriate circle bundles over $M$. Replacing the circle by the group $\mathrm{G}=$ $\mathrm{Sp}(1)$, acting on $\mathbb{H}$ on the right, one obtains the equations of Pidstrigatch-Tyurin [32]. 
Acknowledgements These are expanded notes for a lecture given at Imperial College on 10 February 2012 . Thanks to Simon Donaldson and Thomas Walpuski for many helpful discussions and for their hospitality during my visit. Thanks to Yasha Eliashberg for his explanation of the h-principle, and thanks to the referee and editor for helpful comments on the exposition.

\section{Appendix A: Divergence-free frames}

Let $M$ be a closed connected oriented 3-manifold and $\operatorname{dvol}_{M} \in \Omega^{3}(M)$ be a positive volume form. Denote the set of positive frames by

$$
\mathcal{F}:=\left\{v=\left(v_{1}, v_{2}, v_{3}\right) \in \operatorname{Vect}(M)^{3} \mid \operatorname{dvol}_{M}\left(v_{1}, v_{2}, v_{3}\right)>0\right\}
$$

and the set of divergence-free positive frames by

$$
\mathcal{V}:=\left\{v \in \mathcal{F} \mid d \iota\left(v_{i}\right) \mathrm{dvol}_{M}=0 \text { for } i=1,2,3\right\} .
$$

Given three deRham cohomology classes $a_{1}, a_{2}, a_{3} \in H^{2}(M ; \mathbb{R})$, denote the set of divergencefree positive frames that represent the classes $a_{i}$ by

$$
\mathcal{V}_{a}:=\left\{v \in \mathcal{V} \mid\left[\iota\left(v_{i}\right) \mathrm{dvol}_{M}\right]=a_{i} \text { for } i=1,2,3\right\} .
$$

The following theorem is an application of Gromov's h-principle and is proved in [21, page 182] and [10, Corollary 20.4.3]. Although the result is stated in these references in the weaker form that every frame can be deformed in $\mathcal{F}$ to a divergence-free frame, the proofs give the stronger result stated below (as explained to the author by Yasha Eliashberg).

Theorem A.1 (Gromov) The inclusion $\mathcal{V}_{a} \hookrightarrow \mathcal{F}$ is a homotopy equivalence for all $a_{1}, a_{2}, a_{3} \in H^{2}(M ; \mathbb{R})$, and so is the inclusion $\mathcal{V} \hookrightarrow \mathcal{F}$.

Lemma A.2 (Gromov) Let $V$ be a 3-dimensional real vector space and $S: V \times V \rightarrow V$ be a skew-symmetric bilinear map. Let $\mathcal{R} \subset \operatorname{Hom}(V, \operatorname{End}(V))$ be the set of all linear maps $L: V \rightarrow \operatorname{End}(V)$ such that the map

$$
\Lambda^{2} V \rightarrow V: u \wedge v \mapsto S(u, v)+L(u) v-L(v) u
$$

is a vector space isomorphism. Fix a 2-dimensional linear subspace $E \subset V$ and a linear map $\lambda: E \rightarrow \operatorname{End}(V)$. Define

$$
\mathcal{L}:=\left\{L \in \operatorname{Hom}(V, \operatorname{End}(V))|L|_{E}=\lambda\right\} .
$$

If $\mathcal{L} \cap \mathcal{R}$ is nonempty, then it has two connected components and the convex hull of each connected component of $\mathcal{L} \cap \mathcal{R}$ is equal to $\mathcal{L}$.

Proof The proof is a special case of the argument given by Eliashberg-Mishachev in [10, pages 183/184]. Assume without loss of generality that

$$
V=\mathbb{R}^{3}, \quad E=\left\{x \in \mathbb{R}^{3} \mid x_{1}=0\right\} .
$$

Write $S$ in the form

$$
S(u, v)=: \sum_{i<j} u_{i} v_{j} S_{i j}, \quad S_{i j}=-S_{j i} \in \mathbb{R}^{3}
$$


and write a linear map $L: \mathbb{R}^{3} \rightarrow \operatorname{End}\left(\mathbb{R}^{3}\right)$ in the form

$$
L(u) v=\sum_{i, j=1}^{3} u_{i} v_{j} L_{i j}, \quad L_{i j} \in \mathbb{R}^{3} .
$$

Then $L \in \mathcal{R}$ if and only if

$$
\operatorname{det}\left(S_{23}+L_{23}-L_{32}, S_{31}+L_{31}-L_{13}, S_{12}+L_{12}-L_{21}\right) \neq 0 .
$$

Denote by $\mathcal{R}^{+}$, respectively $\mathcal{R}^{-}$, the set of all $L \in \mathcal{R}$ for which the sign of the determinant in (52) is positive, respectively negative.

Fix a linear map $\lambda: E \rightarrow \operatorname{End}\left(\mathbb{R}^{3}\right)$. This map is determined by the coefficients $L_{i j}$ with $i=2,3$. Thus an element $L \in \mathcal{L} \cap \mathcal{R}$ is determined by the choice of $L_{11}, L_{12}, L_{13}$. If $S_{23}+$ $L_{23}-L_{32}=0$ then the determinant in (52) vanishes for every $L \in \mathcal{L}$ and so $\mathcal{L} \cap \mathcal{R}=\emptyset$. Hence assume $S_{23}+L_{23}-L_{32} \neq 0$. Then $\mathcal{L} \cap \mathcal{R}^{+}$and $\mathcal{L} \cap \mathcal{R}^{-}$are nonempty connected submanifolds of $\mathbb{R}^{3} \times \mathbb{R}^{3} \times \mathbb{R}^{3}$. (Namely, $L_{11}$ is any vector in $\mathbb{R}^{3}, L_{12}$ is required to be in the complement of an affine line, and then $L_{13}$ is required to be in the complement of an affine plane depending smoothly on $L_{12}$.)

Choose $x, y \in \mathbb{R}^{3}$ such that

$$
\operatorname{det}\left(S_{23}+L_{23}-L_{32}, x, y\right)>0 .
$$

Then, for $t>0$ sufficiently large,

$$
\begin{aligned}
& \operatorname{det}\left(S_{23}+L_{23}-L_{32}, S_{31}+t x, S_{12}+t y\right)>0, \\
& \operatorname{det}\left(S_{23}+L_{23}-L_{32}, S_{31}-t x, S_{12}-t y\right)>0 .
\end{aligned}
$$

Given $L \in \mathcal{L}$ choose $L^{\prime}, L^{\prime \prime} \in \mathcal{L}$ such that

$$
\begin{array}{lll}
L_{11}^{\prime}=L_{11}, & L_{12}^{\prime}:=L_{21}+t y, & L_{13}^{\prime}:=L_{31}-t x, \\
L_{11}^{\prime \prime}=L_{11}, & L_{12}^{\prime \prime}:=L_{21}-t y, & L_{13}^{\prime \prime}:=L_{31}+t x .
\end{array}
$$

Then $L^{\prime}, L^{\prime \prime} \in \mathcal{L} \cap \mathcal{R}^{+}$and $L=\frac{1}{2}\left(L^{\prime}+L^{\prime \prime}\right)$. Hence the convex hull of $\mathcal{L} \cap \mathcal{R}^{+}$is equal to $\mathcal{L}$. A similar argument shows that the convex hull of $\mathcal{L} \cap \mathcal{R}^{-}$is also equal to $\mathcal{L}$. This proves Lemma A.2.

Proof of Theorem A.l Fix a Riemannian metric on $M$, let $a \in H^{2}(M ; \mathbb{R})^{3}$, and let $\sigma_{i} \in$ $\Omega^{2}(M)$ be the harmonic representative of $a_{i}, i=1,2,3$. Define

$$
\mathscr{H}_{a}:=\left\{\beta=\left(\beta_{1}, \beta_{2}, \beta_{3}\right) \in \Omega^{1}(M)^{3} \mid \sigma_{i}+d \beta_{i} \text { are linearly independent }\right\} .
$$

Let

$$
\pi_{a}: \mathscr{H}_{a} \rightarrow \mathcal{V}_{a}
$$

be the projection defined by $\pi_{a}(\beta)=v$ for $\beta \in \mathscr{H}_{a}$, where $\iota\left(v_{i}\right) \operatorname{dvol}_{M}:=\sigma_{i}+d \beta_{i}$. This is a homotopy equivalence. A homotopy inverse assigns to $v \in \mathcal{V}_{a}$ the unique co-exact triple of 1 -forms $\beta \in \pi_{a}^{-1}(v)$.

Consider the vector bundle

$$
X:=T^{*} M \oplus T^{*} M \oplus T^{*} M
$$


over $M$ and denote by $X^{(1)}$ the 1 -jet bundle. Use the Riemannian metric on $M$ to identify $X^{(1)}$ with the set of tuples $\left(y, \beta_{1}, \beta_{1}, \beta_{3}, L_{1}, L_{2}, L_{3}\right)$ with $y \in M, \beta_{i} \in T_{y}^{*} M$, and $L_{i} \in \operatorname{Hom}\left(T_{y} M, T_{y}^{*} M\right)$. Denote by

$$
\mathcal{R}_{a} \subset X^{(1)}
$$

the open subset of all $(y, \beta, L) \in X^{(1)}$ such that the 2-forms $\tau_{i} \in \Lambda^{2} T_{y}^{*} M$, defined by

$$
\tau_{i}(u, v):=\sigma_{i}(u, v)+\left\langle L_{i}(u), v\right\rangle-\left\langle L_{i}(v), u\right\rangle, \quad i=1,2,3,
$$

are linearly independent. Denote by $\delta_{a}$ the space of sections of $\mathcal{R}_{a}$. Thus an element of $\oiint_{a}$ is a tuple $(\beta, L)=\left(\beta_{1}, \beta_{2}, \beta_{3}, L_{1}, L_{2}, L_{3}\right)$ with $\beta_{i} \in \Omega^{1}(M)$ and $L_{i} \in \Omega^{1}\left(M, T^{*} M\right)$ such that the 2-forms $\tau_{i} \in \Omega^{2}(M)$, defined by (53) are everywhere linearly independent. Then $\delta_{a}$ is a bundle over $\mathcal{F}$. The projection $\pi_{a}: \delta_{a} \rightarrow \mathcal{F}$ is given by $\pi_{a}(\beta, L)=v$, where $\iota\left(v_{i}\right) \operatorname{dvol}_{M}:=$ $\tau_{i}$ and $\tau_{i}$ is as in (53). This map is a homotopy equivalence. A homotopy inverse of $\pi_{a}$ is the inclusion $\iota_{a}: \mathcal{F} \rightarrow \wp_{a}$ given by $\iota_{a}(v):=(0, L)$, where $L_{i}(u):=\frac{1}{2}\left(\operatorname{dvol}_{M}\left(v_{i}, u, \cdot\right)-\right.$ $\left.\sigma_{i}(u, \cdot)\right)$. Namely, $\pi_{a} \circ \iota_{a}=\mathrm{id}: \mathcal{F} \rightarrow \mathcal{F}$, both maps are linear between open subsets of topological vector spaces, and the kernel of $\pi_{a}$ is the space of tuples $(\beta, L)$ such that each $L_{i}$ is symmetric.

The previous discussion shows that there is a commutative diagram

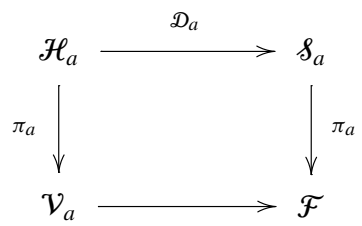

where the vertical maps are homotopy equivalences and the differential operator $\mathscr{D}_{a}: \mathscr{H}_{a} \rightarrow$ $\delta_{a}$ is given by $\mathscr{D}_{a} \beta:=(\beta, \nabla \beta)$. Thus $\mathscr{H}_{a}$ is the space of all sections $\beta$ of $X$ such that $\mathcal{D}_{a} \beta$ satisfies the differential relation $\mathcal{R}_{a}$. By Lemma A.2, $\mathcal{R}_{a}$ is ample in the sense of [10, page 167]. Hence $\mathcal{R}_{a}$ satisfies the h-principle (see [10, Theorem 18.4.1]). In particular, every section of $\mathcal{R}_{a}$ is homotopic, through sections of $\mathcal{R}_{a}$, to a section of the form $(\beta, \nabla \beta)$. Equivalently, every frame $v \in \mathcal{F}$ can be deformed within $\mathcal{F}$ to a divergence-free frame in $\mathcal{V}_{a}$. In fact, by the parametric h-principle, the inclusion $\mathscr{D}_{a}: \mathscr{H}_{a} \rightarrow \wp_{a}$ induces isomorphisms on all homotopy groups, and is therefore a homotopy equivalence (see [10, 6.2.A]). Hence the inclusion $\mathcal{V}_{a} \hookrightarrow \mathcal{F}$ is a homotopy equivalence.

To explain the extension of this result to the inclusion of $\mathcal{V}$ into $\mathcal{F}$, it is convenient to spell out the details of the parametric h-principle in the present setting. Choose a smooth manifold $\Lambda$ and a smooth map $a: \Lambda \rightarrow H^{2}(M ; \mathbb{R})^{3}$. Consider the vector bundle

$$
\widetilde{X}:=\Lambda \times T^{*} M \oplus T^{*} M \oplus T^{*} M \rightarrow \tilde{M}:=\Lambda \times M .
$$

Define $\widetilde{\mathcal{R}} \subset \widetilde{X}^{(1)}$ as the set of tuples $\left(\lambda, y, \beta_{1}, \beta_{2}, \beta_{3}, \widetilde{L}_{1}, \widetilde{L}_{2}, \widetilde{L}_{3}\right)$, with $\lambda \in \Lambda, y \in M, \beta_{i} \in$ $T_{y}^{*} M$, and $\widetilde{L}_{i} \in \operatorname{Hom}\left(T_{\lambda} \Lambda \times T_{y} M, T_{y}^{*} M\right)$, such that the 2 -forms $\tau_{i}=\tau_{\lambda, i} \in \Lambda^{2} T_{y}^{*} M$ in (53) are linearly independent. Here $\sigma_{i}=\sigma_{\lambda, i}$ is the harmonic representative of the class $a_{i}(\lambda)$ and $L_{i} \in \operatorname{Hom}\left(T_{y} M, T_{y}^{*} M\right)$ is the restriction of $\widetilde{L}_{i}$ to $0 \times T_{y} M$. Define the operator $\widetilde{D}$ from sections of $\tilde{X}$ to sections of $\tilde{X}^{(1)}$ as the covariant derivative

$$
\widetilde{D} \beta:=(\beta, \nabla \beta) \text {. }
$$


Let $\widetilde{\&}$ be the space of sections of $\widetilde{\mathcal{R}} \subset \widetilde{X}^{(1)}$ and denote by $\tilde{\mathcal{H}}$ its preimage under $\widetilde{D}$. Thus an element of $\widetilde{\mathscr{H}}$ is a map $\Lambda \rightarrow \Omega^{1}(M)^{3}: \lambda \mapsto \beta_{\lambda}$ such that the 2-forms

$$
\tau_{\lambda, i}:=\sigma_{\lambda, i}+d \beta_{\lambda, i}, \quad i=1,2,3,
$$

are everywhere linearly independent for every $\lambda$. An element of $\tilde{\delta}$ is a smooth section that assigns to $\lambda \in \Lambda$ a tuple

$$
\left(\beta_{\lambda, 1}, \beta_{\lambda, 2}, \beta_{\lambda, 3}, \widetilde{L}_{\lambda, 1}, \widetilde{L}_{\lambda, 2}, \widetilde{L}_{\lambda, 3}\right) \in \Omega^{1}(M)^{3} \times \operatorname{Hom}\left(T_{\lambda} \Lambda, \Omega^{1}\left(M, T^{*} M\right)\right)^{3}
$$

such that the 2-forms $\tau_{\lambda, i} \in \Omega^{2}(M)$, defined by (53), are everywhere linearly independent for every $\lambda \in \Lambda$. As before there is a commutative diagram

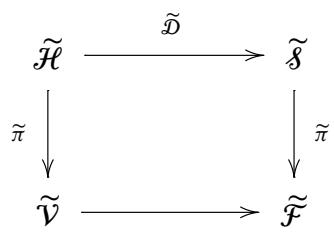

Here $\tilde{\mathcal{V}}$ is the space of maps $\Lambda \rightarrow \mathcal{V}: \lambda \mapsto v_{\lambda}$ such that $v_{\lambda} \in \mathcal{V}_{a(\lambda)}$ and $\widetilde{\mathcal{F}}$ is the space of all smooth maps from $\Lambda$ to $\tilde{\mathcal{F}}$. The projection $\tilde{\pi}: \widetilde{\mathscr{H}} \rightarrow \widetilde{\mathcal{V}}$, respectively $\tilde{\pi}: \widetilde{\mathcal{F}} \rightarrow \widetilde{\mathcal{F}}$, assigns to the section $\lambda \mapsto \beta_{\lambda}$, respectively (55), the section $\lambda \mapsto v_{\lambda}$ with $\iota\left(v_{\lambda, i}\right) \mathrm{dvol}_{M}=\tau_{\lambda, i}$, where $\tau_{\lambda, i}$ is given by (54), respectively (53). Both projections are homotopy equivalences.

By Lemma A.2, the open differential relation $\widetilde{\mathcal{R}}$ is ample. Hence it follows from the h-principle in [10, Theorem 18.4.1] that every smooth map from $\Lambda$ to $\mathcal{F}$ can be deformed within $\widetilde{\mathcal{F}}$ to a smooth map $\Lambda \rightarrow \mathcal{V}: \lambda \mapsto v_{\lambda}$ that satisfies $v_{\lambda} \in \mathcal{V}_{a(\lambda)}$. With $\Lambda=S^{k}$ this implies that the homomorphism $\pi_{k}\left(\mathcal{V}_{a}\right) \rightarrow \pi_{k}(\mathcal{F})$ is surjective for all $a_{1}, a_{2}, a_{3} \in H^{2}(M ; \mathbb{R})$.

The relation $\widetilde{\mathcal{R}}$ also satisfies the relative $h$-principle in [10, 6.2.C]. For the $(k+1)$-ball $\Lambda=B^{k+1}$ with boundary $\partial B^{k+1}=S^{k}$ this means that, if a map $S^{k} \rightarrow \mathcal{V} \subset \mathcal{F}$ extends over $B^{k+1}$ in $\mathcal{F}$, and one chooses any smooth extension of the projection $S^{k} \rightarrow \mathcal{V} \rightarrow H^{2}(M ; \mathbb{R})^{3}$ over $B^{k+1}$, then this extension lifts to a smooth map $B^{k+1} \rightarrow \mathcal{V}$, equal to the given map over the boundary (and homotopic to the given map in $\mathcal{F}$ ). Hence the homomorphism $\pi_{k}(\mathcal{V}) \rightarrow$ $\pi_{k}(\mathcal{F})$ is injective. This proves Theorem A.1.

\section{Appendix B: Self-adjoint Fredholm operators}

This appendix is included for the benefit of the reader. It discusses two well known results about self-adjoint Fredholm operators, that are used in Sect. 2. Lemma B.1 characterizes unbounded self-adjoint Fredholm operators and Lemma B.2 shows that regular crossings are isolated. While Lemma B.2 follows from the Kato selection theorem (see [34, Lemma 4.7]), the proof given below is simpler and more direct.

Let $H$ be a Hilbert space and $V \subset H$ be a dense linear subspace that is a Hilbert space in its own right. Suppose that the inclusion $V \hookrightarrow H$ is a compact operator. Denote the inner product on $H$ by $\langle\cdot, \cdot\rangle$, the norm on $H$ by $\|x\|_{H}:=\sqrt{\langle x, x\rangle}$ for $x \in H$, and the norm on $V$ by $\|x\|_{V}$ for $x \in V$. Let $\&$ be the space of symmetric bounded linear operators $A: V \rightarrow H$ and $\mathscr{D} \subset \&$ be the subset of self-adjoint operators. Thus a bounded linear operator $D: V \rightarrow H$ 
is an element of $\mathscr{D}$ if and only if $\langle D x, \xi\rangle=\langle x, D \xi\rangle$ for all $x, \xi \in V$ and, for every $x \in H$, the following holds

$$
\sup _{0 \neq \xi \in V} \frac{|\langle x, D \xi\rangle|}{\|\xi\|_{H}}<\infty \quad \Longleftrightarrow \quad x \in V .
$$

Every $D \in \mathscr{D}$ is a Fredholm operator of index zero and regular crossings of differentiable paths $\mathbb{R} \rightarrow \mathscr{D}: s \mapsto D(s)$ are isolated. Proofs of these well known observations are included here for completeness of the exposition.

Lemma B.1 Let $D \in$ \&. Then the following are equivalent.

(i) $D \in \mathscr{D}$.

(ii) (im $D)^{\perp} \subset V$ and there is a constant $c>0$ such that, for all $x \in V$,

$$
\|x\|_{V} \leq c\left(\|D x\|_{H}+\|x\|_{H}\right) .
$$

(iii) $D$ is a Fredholm operator of index zero.

In particular, $\mathscr{D}$ is an open subset of $\&$ in the norm topology.

Proof We prove that (i) implies (ii). Assume $D \in \mathscr{D}$. By (56) (im $D)^{\perp} \subset V$. We show that the graph of $D$ is a closed subspace of $H \times H$. Let $x_{n} \in V$ and $x, y \in H$ be such that $\lim _{n \rightarrow \infty}\left\|x-x_{n}\right\|_{H}=0$ and $\lim _{n \rightarrow \infty}\left\|y-D x_{n}\right\|_{H}=0$. Then $\langle x, D \xi\rangle=\lim _{n \rightarrow \infty}\left\langle x_{n}, D \xi\right\rangle=$ $\lim _{n \rightarrow \infty}\left\langle D x_{n}, \xi\right\rangle=\langle y, \xi\rangle$ for $\xi \in V$. Hence $x \in V$ by (56) and, since $D$ is symmetric, it follows that $D x=y$. Thus $D$ has a closed graph. Now $V \rightarrow \operatorname{graph}(D): x \mapsto(x, A x)$ is a bijective bounded linear operator and so has a bounded inverse. This proves (57).

We prove that (ii) implies (iii). Since $V \hookrightarrow H$ is a compact operator, it follows from (57) that $D$ has a finite-dimensional kernel and a closed image (see [27, Lemma A.1.1]). Since (im $D)^{\perp} \subset V$ and $D$ is symmetric, it follows that (im $\left.D\right)^{\perp}=\operatorname{ker} D$. Hence dim coker $D=$ dim ker $D$.

We prove that (iii) implies (i). Let $D \in \&$ be a Fredholm operator of index zero. Then $D$ has a finite-dimensional kernel and a closed image. Since $D$ is symmetric, $\operatorname{ker} D \subset$ $(\text { im } D)^{\perp}$. Since $D$ has Fredholm index zero, $\operatorname{ker} D=(\operatorname{im} D)^{\perp}$ and hence im $D=(\operatorname{ker} D)^{\perp}$. Now let $x \in H$ and suppose that there is a constant $c$ such that $|\langle x, D \xi\rangle| \leq c\|\xi\|_{H}$ for every $\xi \in V$. By the Riesz representation theorem, there exists an element $y \in H$ such that $\langle x, D \xi\rangle=\langle y, \xi\rangle$ for $\xi \in V$. Choose $y_{0} \in \operatorname{ker} D$ such that $y-y_{0} \perp \operatorname{ker} D$. Then $y-y_{0} \in$ im $D$. Choose $x_{1} \in V$ such that $D x_{1}=y-y_{0}$. Then $\left\langle x-x_{1}, D \xi\right\rangle=\langle y, \xi\rangle-\left\langle D x_{1}, \xi\right\rangle=$ $\left\langle y_{0}, \xi\right\rangle$ for every $\xi \in V$. Given $\xi \in V$ choose $\xi_{0} \in \operatorname{ker} D$ such that $\xi-\xi_{0} \perp \operatorname{ker} D$. Then

$$
\left\langle x-x_{1}, D \xi\right\rangle=\left\langle x-x_{1}, D\left(\xi-\xi_{0}\right)\right\rangle=\left\langle y_{0}, \xi-\xi_{0}\right\rangle=0 .
$$

Hence $x-x_{1} \in(\operatorname{im} D)^{\perp}=\operatorname{ker} D \subset V$ and hence $x \in V$.

Since (i) and (iii) are equivalent it follows from the perturbation theory for Fredholm operators (see [27, Theorem A.1.5]) that $\mathscr{D}$ is an open subset of $\&$ with respect to the norm topology. This proves Lemma B.1.

Let $I \subset \mathbb{R}$ be an open interval and $I \rightarrow \mathscr{D}: s \mapsto D(s)$ be a continuous path with respect to the norm topology on $\mathscr{D}$. The path is called weakly differentiable if the map $I \rightarrow \mathbb{R}: s \mapsto\langle x, D(s) \xi\rangle$ is differentiable for every $x \in H$ and every $\xi \in V$. A crossing is 
an element $s \in I$ such that $D(s)$ has a nontrivial kernel. A crossing $s \in I$ is called regular if the quadratic form

$$
\Gamma_{s}: \operatorname{ker} D(s) \rightarrow \mathbb{R}, \quad \Gamma_{s}(\xi):=\langle\xi, \dot{D}(s) \xi\rangle
$$

is nondegenerate.

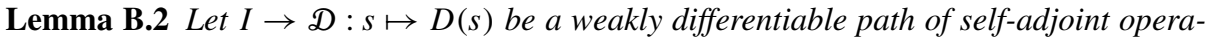
tors and let $s_{0} \in I$ be a regular crossing. Then there is a $\delta>0$ such that $D(s): V \rightarrow H$ is bijective for every $s \in I$ with $0<\left|s-s_{0}\right|<\delta$.

Proof Assume without loss of generality that $s_{0}=0$. By Lemma B.1 there is a constant $c>0$ such that

$$
\|x\|_{V} \leq c\left(\|D(s) x\|_{H}+\|x\|_{H}\right)
$$

for every $x \in V$ and every $s$ in some neighborhood of zero. Shrinking $I$, if necessary, we may assume that (58) holds for every $s \in I$.

Assume, by contradiction, that there is a sequence $s_{n} \in I$ such that $s_{n} \rightarrow 0$ and $D\left(s_{n}\right)$ is not injective for every $n$. Then there is a sequence $x_{n} \in V$ such that $D\left(s_{n}\right) x_{n}=0$ and $\left\|x_{n}\right\|_{H}=1$. Thus $\left\|x_{n}\right\|_{V} \leq c$ by (58). Passing to a subsequence we may assume that $x_{n}$ converges in $H$ to $x_{0}$. Then $\left\|x_{0}\right\|_{H}=1$ and $\left\langle x_{0}, D(0) \xi\right\rangle=\lim _{n \rightarrow \infty}\left\langle x_{n}, D\left(s_{n}\right) \xi\right\rangle=0$ for $\xi \in V$. Hence $x_{0} \in \operatorname{ker} D(0)$. Moreover, for every $\xi \in \operatorname{ker} D(0)$, the sequence $D\left(s_{n}\right) \xi / s_{n}$ converges weakly to $\dot{D}(0) \xi$ and is therefore bounded, so

$$
\left\langle\dot{D}(0) \xi, x_{0}\right\rangle=\lim _{n \rightarrow \infty}\left\langle\frac{D\left(s_{n}\right) \xi}{s_{n}}, x_{0}\right\rangle=\lim _{n \rightarrow \infty}\left\langle\frac{D\left(s_{n}\right) \xi}{s_{n}}, x_{n}\right\rangle=0 .
$$

This contradicts the nondegeneracy of $\Gamma_{0}$ and proves Lemma B.2.

Let $I$ be a compact interval and $I \rightarrow \mathscr{D}: s \mapsto D(s)$ be a weakly differentiable path with only regular crossings such that $D(s)$ is bijective at the endpoints of $I$. The spectral flow is the sum of the signatures of the crossing forms $\Gamma_{s}$ over all crossings. It is invariant under homotopy with fixed endpoints and is additive under catenation. (See [34] for an exposition.)

\section{References}

1. Atiyah, M.F., Patodi, V.K., Singer, I.M.: Spectral asymmetry and Riemannian geometry III. Math. Proc. Camb. Philos. Soc. 79, 71-99 (1976)

2. Cieliebak, K., Gaio, A.R., Salamon, D.A.: J-holomorphic curves, moment maps, and invariants of Hamiltonian group actions. Int. Math. Res. Not. 10, 831-882 (2000)

3. Cieliebak, K., Gaio, A.R., Mundet i Riera, I., Salamon, D.A.: The symplectic vortex equations and invariants of Hamiltonian group actions. J. Symplectic Geom. 1, 543-645 (2002)

4. Conley, C., Zehnder, E.: The Birkhoff-Lewis fixed point theorem and a conjecture of V.I. Arnold. Invent. Math. 73, 33-49 (1983)

5. Donaldson, S.K., Kronheimer, P.B.: The Geometry of Four-Manifolds. Oxford University Press, Oxford (1990)

6. Donaldson, S.K., Thomas, R.P.: Gauge theory in higher dimensions. In: The Geometric Universe, Oxford, 1996, pp. 31-47. Oxford University Press, Oxford (1998)

7. Donaldson, S.K., Segal, E.: Gauge theory in higher dimensions II. Preprint (2009)

8. Dostoglou, S., Salamon, D.A.: Self-dual instantons and holomorphic curves. Ann. Math. 139, 581-640 (1994) 
9. Ebert, J.: A vanishing theorem for Characteristic classes of odd-dimensional manifold bundles. Preprint (2010) arXiv:0902.4719v3

10. Eliashberg, Y., Mishachev, N.: Introduction to the h-Principle. AMS, Providance (2002)

11. Floer, A.: Morse theory for Lagrangian intersections. J. Differ. Geom. 28, 513-547 (1988)

12. Floer, A.: The unregularized gradient flow of the symplectic action. Commun. Pure Appl. Math. 41, 775-813 (1988)

13. Floer, A.: Symplectic fixed points and holomorphic spheres. Commun. Math. Phys. 120, 575-611 (1989)

14. Floer, A.: An instanton invariant of 3-manifolds. Commun. Math. Phys. 118, 215-240 (1989)

15. Frauenfelder, U.: The Arnold-Givental conjecture and moment Floer homology. Int Math Res Not 21792269 (2004)

16. Fueter, R.: Analytische Funktionen einer Quaternionenvariablen. Comment. Math. Helv. 4, 9-20 (1932)

17. Fueter, R.: Die Theorie der regulären Funktionen einer Quaternionenvariablen. C. R. 1, 75-91 (1937). ICM Oslo (1936)

18. Gaio, A.R., Salamon, D.A.: Gromov-Witten invariants of symplectic quotients and adiabatic limits. J. Symplectic Geom. 3, 55-159 (2005)

19. Ginzburg, V., Hein, D.: Hyperkähler Arnold conjecture and its generalizations. Int. Math. Res. Not. 5368 (2010)

20. Ginzburg, V., Hein, D.: Arnold conjecture for Clifford symplectic pencils. To appear in Isr. J. Math. (2013)

21. Gromov, M.: Partial Differential Relations. Springer, Berlin (1986)

22. Haydys, A.: Nonlinear Dirac operator and quaternionic analysis. Commun. Math. Phys. 281, 251-261 (2008)

23. Hohloch, S., Noetzel, G., Salamon, D.A.: Hypercontact structures and Floer homology. Geom. Topol. 13, 2543-2617 (2009)

24. Hohloch, S., Noetzel, G., Salamon, D.A.: Floer homology groups in hyperkähler geometry. In: Abreu, M., Lalonde, F., Polterovich, L. (eds.) New Perspectives and Challenges in Symplectic Field Theory. CRM, vol. 49, pp. 251-261. AMS, Providance (2009)

25. Joyce, D.: Compact Manifolds with Special Holonomy. Oxford University Press, Oxford (2000)

26. Kronheimer, P., Mrowka, T.: Monopoles and Three Manifolds. CUP, Cambridge (2007)

27. McDuff, D., Salamon, D.A.: J-Holomorphic Curves and Symplectic Topology, 2nd edn. AMS Colloquium Publications, vol. 52. AMS, Providance (2012)

28. Mundet i Riera, I.: Hamiltonian Gromov-Witten invariants. Topology 42, 525-553 (2003)

29. Ott, A.: Removal of singularities and Gromov compactness for symplectic vortices. J. Symplectic Geom.

30. Ott, A., Ziltener, F.: Gauged Gromov-Witten invariants for monotone symplectic manifolds

31. Pidstrigatch, V.Y.: Hyperkähler manifolds and the Seiberg-Witten equations. Proc. Steklov Inst. Math. 246, 249-262 (2004)

32. Pidstrigatch, V.Y., Tyurin, A.N.: Localization of Donaldson invariants along the Seiberg-Witten basic classes. Preprint (1995). arXiv:dg-ga/9507004

33. Pozniak, M.: Floer homology, Novikov rings and clean intersections. Eliashberg, Y., Fuchs, D., Ratiu, T., Weinstein, A. (eds.) North. Calif. Symp. Geo. Seminar. UCB. AMS, Providance (1999)

34. Robbin, J.W., Salamon, D.A.: The spectral flow and the Maslov index. Bull. Lond. Math. Soc. 27, 1-33 (1995)

35. Salamon, D.A., Walpuski, T.: Notes on the octonians. Preprint (2010)

36. Taubes, C.H.: Nonlinear generalizations of a 3-manifold's Dirac operator. In: Trends in Mathematical Physics, Knoxville, TN, 1998. AMS/IP Stud. Adv. Math., vol. 13, pp. 475-486 (1999)

37. Walpuski, T.: Private communication. February (2011)

38. Walpuski, T.: G2-instantons on generalized Kummer constructions. Preprint (2011). arXiv:1109.6609

39. Walpuski, T.: G2-instantons, associative submanifolds and Fueter sections. Preprint (2012). arXiv:1205. 5350 